\title{
Stackelberg Game for Cognitive Radio Networks With MIMO and Distributed Interference Alignment
}

\author{
Yi Xu, Student Member, IEEE, and Shiwen Mao, Senior Member, IEEE
}

\begin{abstract}
In this paper, we investigate the problem of spectrum leasing in cognitive radio (CR) networks, while incorporating two advanced physical-layer technologies, i.e., multiple-input multipleoutput (MIMO) and distributed interference alignment. We present a cooperative spectrum leasing scheme for primary and secondary users to balance the tension between data transmission and revenue collection/payment. A Stackelberg game is formulated, where the primary user is the leader, and secondary users are followers. With backward induction, we derive the optimal strategies for primary and secondary users that can achieve the unique Stackelberg equilibrium, where no player can gain by a unilaterally changing strategy. We find that spectrum leasing is always beneficial to enhancing the utilities of primary and secondary users. The proposed scheme outperforms a no-spectrum-leasing scheme and a cooperative scheme presented in the literature with considerable gains, which demonstrate the benefits of spectrum leasing and distributed interference alignment and validates the efficacy of the proposed scheme.
\end{abstract}

Index Terms-Cognitive radio (CR) networks, distributed interference alignment, interference alignment, multiple-input multiple-output (MIMO), Stackelberg game.

\section{INTRODUCTION}

\section{A. Background and Motivation}

D UE TO the tremendous increase in wireless data traffic, a usable radio spectrum is quickly being depleted. However, according to the Federal Communications Commission report [2], while some licensed bands are overcrowded, many others are underutilized. Under the traditional fixed spectrum allocation policy, when licensed users (or primary users) are not active, the channels assigned to them are wasted (termed as spectrum opportunities). Cognitive radios (CRs) are proposed as a new wireless paradigm for exploiting such spectrum opportunities, to enable flexible and efficient access to a radio spectrum [3]. In CR networks, unlicensed users (or secondary users) are allowed to access the licensed band opportunistically, whereas primary users gain by collecting revenue for spectrum leasing.

Such a CR paradigm has been shown to have high potentials for enhancing spectrum efficiency [4]. As significant advances

Manuscript received April 28, 2013; revised July 12, 2013; accepted August 23, 2013. Date of publication August 28, 2013; date of current version February 12, 2014. This work was supported in part by the U.S. National Science Foundation under Grant CNS-0953513 and Grant CNS-1247955. This paper was presented in part at the 22nd IEEE International Conference on Computer Communications and Networks, Nassau, Bahamas, July-August 2013. The review of this paper was coordinated by Prof. Y. Cheng.

The authors are with the Department of Electrical and Computer Engineering, Auburn University, Auburn, AL 36849 USA (e-mail: yzx0010@ auburn.edu; smao@ieee.org).

Color versions of one or more of the figures in this paper are available online at http://ieeexplore.ieee.org.

Digital Object Identifier 10.1109/TVT.2013.2279761 are made in many aspects of $\mathrm{CR}$ research, such as spectrum sensing and dynamic spectrum access, it is also desirable to incorporate advanced physical-layer techniques into CR networks. One of such techniques is multiple-input multiple-output (MIMO), which can be used to reduce the bit error rate, transmit more packets, or strengthen the signal-to-interferenceand-noise ratio. In the past decade, MIMO has evolved from a theoretic concept to a technology that can be widely used in practice [5]. It is desirable to exploit MIMO for enhanced primary and secondary transmissions.

The second physical-layer technology is interference alignment, which is a significant breakthrough that exploits interference in interference-limited wireless networks [6]. Traditionally, if interference is small, it is simply treated as background noise; if interference is large, it can be decoded first and then removed from the received signal (i.e., interference cancelation); if interference is comparable to the desired signal, we usually try to avoid it by orthogonalizing the channels or adopting a medium access control mechanism. Unlike traditional approaches, interference alignment casts interference to half of the received signal space to achieve a normalized degree of freedom (DoF) of $K / 2$, where $K$ is the number of interfering users. ${ }^{1}$ Since an interference-free channel only has a normalized DoF of 1 , substantial system throughput gain can be achieved with interference alignment when $K$ is large. For interference alignment, a strong requirement is the availability of global channel state information (CSI) at every node. To relax this requirement, distributed interference alignment is investigated, and an iterative algorithm is proposed in [7] to achieve interference alignment with local CSI.

\section{B. Approach}

In this paper, we investigate how to incorporate these two advanced physical-layer technologies, i.e., MIMO and distributed interference alignment, in CR networks. The CR network consists of a primary user and multiple secondary users, each with $N$ antennas. Time is divided into time slots of equal length with a normalized length. The primary user has some data to send and requires a certain nonzero data rate in each time slot. It also leases a spectrum to secondary users for more revenue. Secondary users pay the primary user for data transmission in the time slot [1].

A key observation is that the licensed users usually have a finite amount of data to send. After a period of high rate

\footnotetext{
${ }^{1}$ If the capacity can be written as $C(\mathrm{SNR})=d \log (\mathrm{SNR})+o(\log (\mathrm{SNR}))$ then the channel is said to have a DoF of $d$ [6].
} 
transmission, they might be interested in leasing the spectrum to unlicensed users so that revenue can be collected. On the other hand, the unlicensed users desire the opportunities for data transmission if the associated cost is acceptable. Therefore, in the proposed cooperative spectrum leasing scheme, the primary user divides each time slot into three phases: i) In Phase I, only the primary user transmits with MIMO; ii) in Phase II, the primary user and a selected set of secondary users simultaneously transmit using distributed interference alignment; and iii) in Phase III, only the selected secondary users transmit with distributed interference alignment. The primary user decides the division of the three phases, selects the set of secondary users for spectrum leasing, and collects a revenue from the selected secondary users proportional to their transmit powers (or data rates).

We find that such a cooperative spectrum leasing framework fits well with the Stackelberg game theory [19]. In the formulated Stackelberg game, the primary user is the leader, and the secondary users are followers. The leader decides the division of a time slot into three phases and the selection of followers, aiming to balance its own data transmission and revenue collection by leasing a spectrum. Once the leaders' decisions are made, a follower can choose a transmit power (and thus the corresponding data rate) based on how much it is willing to pay. We define the Stackelberg equilibrium, where neither the primary user nor any secondary user could gain by a unilateral change of strategy. We present a rigorous analysis with the backward induction method [19] and derive the unique Stackelberg equilibrium for the cooperative spectrum leasing game.

We find that the most desirable scenario for secondary users is to have only Phase III in the time slot with only three players. The strategy for the primary user depends on the number of secondary users. With more than $2 N-2$ secondary users, exactly $2 N-2$ secondary users will be selected, each having one interference-free channel, and there will be only Phase II in the time slot. With less than $2 N-2$ secondary users, all secondary users will be selected, and there will be only Phases II and III in the time slot. Therefore, spectrum leasing is always helpful in increasing the utilities of both the primary and secondary users. In the simulation study, we first compare the proposed scheme with a scheme without spectrum leasing to demonstrate the benefits of spectrum leasing. We then compare the proposed scheme with the cooperative scheme presented in [8] to demonstrate the efficacy of distributed interference alignment. Significant performance gains are achieved by the proposed scheme in these simulations.

\section{Related Work}

This paper is closely related to the research on CR networks. (For a general survey of CRs, see [4].) In a CR network, the primary user is either aware or unaware of the existence of secondary users. This paper falls under the first category. The primary user not only is aware of the existence of secondary users but also knows the impact of the rules on secondaryuser behavior. Most of the previous work, such as [8]-[12], only considered the single-antenna case, whereas we con- sider multiple antennas and exploit multiplexing gain in this paper.

This paper is also related to the research on interference alignment. In [6], Cadambe and Jafar introduced the interference alignment technique. The significance of their work is that, by adopting interference alignment, the system is no longer interference limited. With symbol extension, the system could achieve a normalized DoF of $K / 2$. Another important issue, i.e., the feasibility condition, was investigated in [13] for structureless generic wireless channels. For wireless channels with a structure, such as diagonal channels, our recent paper [14] investigated the application of interference alignment in multiuser orthogonal frequency-division multiplexing networks. To address the concern on the global CSI requirement, a distributed interference alignment algorithm was proposed in [7], which only requires local CSI. In [15], interference alignment and cancelation were integrated to enhance the throughput of MIMO Wi-Fi networks. In [16], Li et al. proposed a general algorithm for the multihop mesh networks. This work was motived by these interesting papers. However, much of the related work mainly focused on physical-layer issues. This paper considers how to adopt distributed interference alignment in a MIMO CR network with a novel Stackelberg-game-based approach.

Recent works, i.e., [17] and [18], have considered the problem of incorporating interference alignment in $\mathrm{CR}$ networks under the same one-primary-user-multiple-secondary-user scenario. In [17], Amir et al. characterized the achievable DoF for the secondary users and an iterative algorithm to achieve the DoF. In [18], Zhou et al. optimized both the precoding vectors and power allocation to enhance the rates of secondary users, where a gradient method is used. However, the prior work does not take into consideration the fact that the primary user has a finite amount of data to send in practice. This paper mainly considers how to use distributed interference alignment in the MIMO CR network and focuses on the case of finite demand of the primary user.

\section{Organization}

The remainder of this paper is organized as follows: In Section II, we introduce the preliminaries and system model. We define the Stackelberg game in Section III and derive the Stackelberg equilibrium in Section IV. Simulation results are presented in Section V. Section VI concludes this paper. The notations used in this paper are summarized in Table I.

\section{Preliminaries And System Model}

\section{A. MIMO and Distributed Interference Alignment}

This paper is closely related to MIMO and distributed interference alignment. We briefly review the preliminaries in this section. More details can be found in [5] and [7]. (For recent developments in MIMO techniques, see [20]-[22].)

1) MIMO Capacity Basics: With the advance of antenna technology, it is now feasible to equip wireless devices with multiple antennas. In general, three types of performance gains 
TABLE I

NOtATION TABLE

\begin{tabular}{rl}
\hline Symbol & Definition \\
\hline $\mathbf{A}$ & Matrix $\mathbf{A}$ \\
$\vec{a}$ & Vector $\vec{a}$ \\
$a$ & Scalar a \\
$\overleftarrow{\mathbf{A}}$ & Matrix $\overleftarrow{\mathbf{A}}$ of the reverse link \\
$\overleftarrow{\vec{a}}$ & Vector $\overleftrightarrow{\vec{a}}$ of the reverse link \\
$\overleftarrow{a}$ & Scalar $\overleftarrow{a}$ of the reverse link \\
$\lfloor a\rfloor$ & Floor function \\
$(\mathbf{A})^{H}$ & Transpose conjugate of matrix $\mathbf{A}$ \\
$C$ & Capacity \\
$d$ & Degree of Freedom \\
$p_{i}$ & Power allocated to the $i$-th channel \\
$P_{i}$ & Total power of $i$-th the secondary user \\
$\vec{P}_{-i}$ & Power of users except user $i$ \\
$P$ & Total power of the primary user \\
$\sigma_{i}$ & $i$-th largest singular value of channel \\
$h$ & Channel state \\
$N_{0}$ & Noise power spectral density \\
$\mathbf{Q}_{k}$ & Interference matrix at the $k$-th receiver \\
$\mathbf{H}_{i j}$ & Channel between transmitter $i$ and receiver $j$ \\
$\mathbf{V}_{i}$ & Precoding matrix of transmitter $i$ \\
$\mathbf{U}_{k}$ & Interference Cancellation matrix of receiver $k$ \\
$\nu_{i}[\mathbf{A}]$ & $i$-th smallest eigenvalue's corresponding eigenvector \\
$R_{P}^{I}$ & Achievable rate of the primary user at Phase I \\
$R_{S_{i}}^{I I}$ & Achievable rate of the $i$-th secondary user at Phase II \\
$d_{I I}$ & Degree of Freedom at Phase II \\
$w_{P}$ & Weight of the primary user \\
$w_{S}$ & Weight of the secondary user \\
$C_{0}$ & Unit price set by the primary user \\
$N$ & Number of antennas \\
$M$ & Number of antennas \\
$K$ & Number of SUs selected to participate in the game \\
$K_{T}$ & Total number of secondary users \\
$U_{P}$ & Utility of the primary user \\
$U_{S_{i}}$ & Utility of the secondary user \\
$U_{P}^{*}$ & Maximum of $U_{P}$ \\
$U_{S_{i}}^{*}$ & Maximum of $U_{S_{i}}$ \\
$\alpha$ & Total length of Phase I and Phase II \\
$\beta$ & Ratio of the length of Phase I to Phase I plus Phase II \\
\hline &
\end{tabular}

can be achieved with MIMO, namely, diversity gain, multiplexing gain, and antenna gain.

For a MIMO system with $N \geq 2$ antennas, assume that the CSI $\mathbf{H}$ is known at the transmitter. Since the MIMO channel can be decomposed into $d$ parallel channels, the channel capacity is given by [23]

$$
C=\max _{p_{i}: \sum_{i} p_{i} \leq P} \sum_{i=1}^{d} \log \left(1+\frac{\sigma_{i}^{2} p_{i}}{N_{0}}\right)
$$

where $P$ denotes the total transmitter power limit, $p_{i}$ is the power allocated to the $i$ th parallel channel, $\sigma_{i}^{2}=\lambda_{i}$, and $\lambda_{i}$ is the $i$ th largest eigenvalue of matrix $\mathbf{H H}^{H}$. Note that bandwidth is normalized throughout this paper.

In the high-SNR region, equal power allocation is shown to be suboptimal but is easier for mathematical modeling than waterfilling. When the transmit power is $P / d$ for each parallel channel, the total capacity can be approximated as

$$
\begin{aligned}
C \approx \sum_{i=1}^{d} \log \left(1+\frac{P \sigma_{i}^{2}}{d N_{0}}\right) & \approx \sum_{i=1}^{d} \log \left(\frac{P \sigma_{i}^{2}}{d N_{0}}\right) \\
= & d \log (\mathrm{SNR})+\sum_{i=1}^{d} \log \left(\frac{\sigma_{i}^{2}}{d}\right) .
\end{aligned}
$$

The second item in (2) is negligible when the SNR is high. We thus ignore this term in the following analysis.

2) Distributed Interference Alignment: The basic idea of interference alignment is to cast the interference to no more than half of the received signal space and leave the other half clean and recognizable. If there are $K$ users, in total, $K / 2$ normalized DoF could be achieved. The system throughput can be greatly enhanced when $K$ is large. For $K=0$ and 1, there is no interference; for $K=2$, the normalized DoF is 1 , which is another trivial case. Therefore, we only consider the case where the number of interfering nodes $K$ satisfies $K \geq 3$. It is worth noting that, to align interference perfectly, global CSI is required at every participating node. To overcome this challenge, an iterative distributed interference alignment algorithm was proposed in [7], which only requires local CSI at each interfering node. By utilizing the reciprocity of wireless networks, it works as follows [7]. Thus

First, compute the interference covariance at each receiver.

$$
\mathbf{Q}_{k}=\sum_{j=1, j \neq k}^{K} \frac{P_{j}}{d_{j}} \mathbf{H}_{j k} \mathbf{V}_{j} \mathbf{V}_{j}^{H} \mathbf{H}_{j k}^{H}
$$

where $P_{j}$ is the total transmitting power of user $j, \mathbf{V}_{j}$ is the precoding matrix at transmitter $j$, and $\mathbf{H}_{j k}$ is the channel gain from transmitter $j$ to receiver $k$. Minimizing the interference leakage at each receiver, the interference cancelation matrix $\mathbf{U}_{k}$ is given as

$$
\vec{u}_{k_{i}}=\nu_{i}\left[\mathbf{Q}_{k}\right], \quad i=1, \ldots, d
$$

where $\vec{u}_{k_{i}}$ is the $i$ th column of $\mathbf{U}_{k}$, and $\nu_{i}\left[\mathbf{Q}_{k}\right]$ is the $i$ th smallest eigenvalue's corresponding eigenvector.

Then, reverse the direction of communication, and let $\overleftarrow{\mathbf{V}}_{k}=$ $\mathbf{U}_{k}$. The interference at the reverse link's receiver is

$$
\overleftarrow{\mathbf{Q}}_{k}=\sum_{j=1, j \neq k}^{K} \frac{\overleftarrow{P}_{j}}{d_{j}} \overleftarrow{\mathbf{H}}_{j k} \overleftarrow{\mathbf{V}}_{j} \overleftarrow{\mathbf{V}}_{j}^{H} \overleftarrow{\mathbf{H}}_{j k}^{H}
$$

Minimizing the interference leakage at each receiver of the reverse link, the interference cancelation matrix is given as

$$
\overleftarrow{\vec{u}}_{k_{i}}=\nu_{i}\left[\overleftarrow{\mathbf{Q}}_{k}\right], \quad i=1, \ldots, d
$$

Then, reverse the direction again, and let $\mathbf{V}_{k}=\overleftarrow{\mathbf{U}}_{k}$. These steps are repeated until convergence is achieved.

The general feasibility condition for interference alignment is given by

$$
\begin{array}{r}
\mathbf{U}_{k}^{H} \mathbf{H}_{j k} \mathbf{V}_{j}=0, \quad \text { for } j \neq k \\
\operatorname{rank}\left(\mathbf{U}_{k}^{H} \mathbf{H}_{k k} \mathbf{V}_{k}\right)=d_{k}, \quad \text { for all } k .
\end{array}
$$

In [13], a system is called proper if it satisfies the following condition:

$$
d \leq 2 N /(K+1) .
$$

Since distributed interference alignment should also satisfy the conditions given in (7) and (8), to simplify the discussion, 


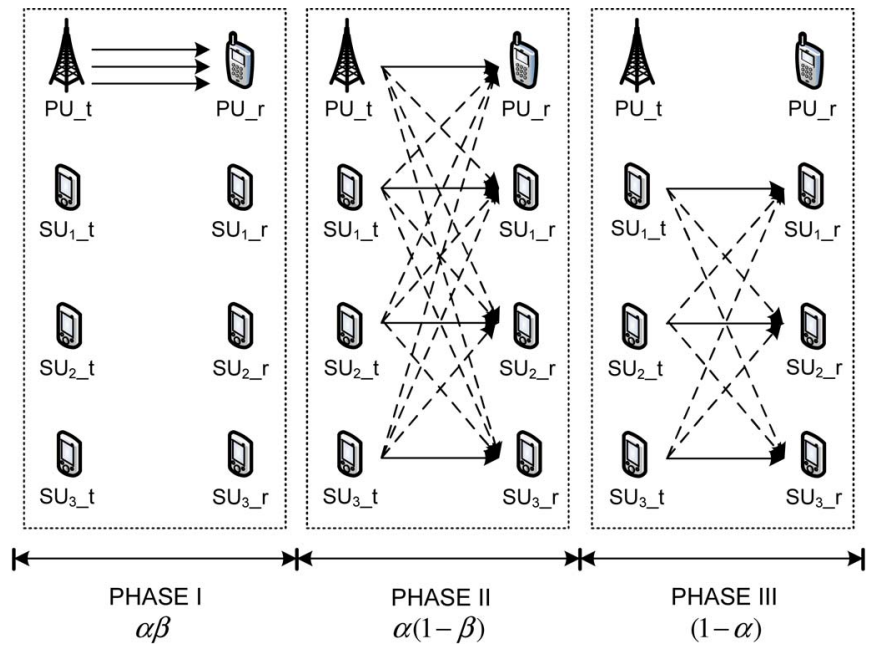

Fig. 1. Three-phase operation of the MIMO CR network with distributed interference alignment.

we consider a proper system to be feasible for distributed interference alignment.

\section{B. System Model and Assumptions}

The MIMO CR network is shown in Fig. 1. There are one primary user and $K_{T}$ secondary users sharing the licensed spectrum, each with $N$ antennas. We consider a time-slotted system, where each time slot is normalized to 1 unit in length and is divided into three phases, with lengths $\alpha \beta, \alpha(1-\beta)$, and $(1-\alpha)$, respectively, for fractions $0 \leq \alpha \leq 1$ and $0 \leq \beta \leq 1$.

In Phase I, the primary user transmits its packets at the highest rate using MIMO, and the secondary users remain silent. The DoF for the primary user is $d_{I}=N$. The achievable rate of the primary user in Phase I is

$$
R_{P}^{I}=d_{I} \log (\mathrm{SNR})
$$

where SNR is assumed to be constant during a time slot.

We assume that the primary user always has a finite amount of packets to send in each time slot. After a period of high data rate transmission (with length $\alpha \beta$ ), the primary user has the incentive to lease the spectrum to secondary users to increase its utility, by collecting revenue from selected secondary users (but with a lower data rate for itself). In Phase II, the primary user and $K \in\left[0, K_{T}\right]$ selected secondary users simultaneously transmit using distributed interference alignment, with a DoF of $d_{I I}=\lfloor(2 N / K+2)\rfloor$. A selected secondary user makes payments that are proportional to its transmit power (i.e., its data rate), and the primary user collects payments from all selected secondary users. The achievable rate of the primary user in Phase II is

$$
R_{P}^{I I}=d_{I I} \log (\mathrm{SNR}) .
$$

The achievable rate of secondary user $S_{i}$ in Phase II is

$$
R_{S_{i}}^{I I}=d_{I I} \log \left(\mathrm{SNR}_{i}\right)
$$

where $\mathrm{SNR}_{i}=P_{i} / N_{0}$ is the SNR for each selected secondary user, which is assumed to be constant in a time slot.
In Phase III, the primary user stops its transmission and leases the spectrum to selected secondary users, which transmit using distributed interference alignment with $d_{I I I}=$ $\lfloor(2 N / K+1)\rfloor$. In Phase III, the achievable rate of secondary user $S_{i}$ is

$$
R_{S_{i}}^{I I I}=d_{I I I} \log \left(\mathrm{SNR}_{i}\right) .
$$

As in prior work [12], [24], we assume a common control channel for the primary user and secondary users to exchange precoding and interference cancelation matrices, weight factor information, and fractions $\alpha$ and $\beta$. Channel estimation is completed before data transmissions.

\section{STACKELBERG GAME FORMULATION}

In the MIMO CR network, the primary user decides the division of a time slot into three phases (some could be of zero length if $\alpha$ or $\beta$ is set to zero) and selection of secondary users, while balancing its own data transmission and revenue collection by leasing a spectrum. Once decisions are made by the primary user, a secondary user can choose a transmit power (and the corresponding data rate) based on how much it is willing to pay. Such interactions fit perfectly with the Stackelberg game model [19].

\section{A. Stackelberg Game Formulation}

Here, we formulate a Stackelberg game for the MIMO CR network with distributed interference alignment. The primary user is the leader, and the secondary users are followers. The strategy of the primary user is given by

$$
\mathcal{S}_{P}=\left\{\alpha, \beta, K \mid 0 \leq \alpha \leq 1,0 \leq \beta \leq 1,3 \leq K \leq K_{T}\right\}
$$

where $K_{T}$ is the total number of secondary users in the system.

The secondary-user strategy is to find a transmit power $P_{i}$. Thus

$$
\mathcal{S}_{S_{i}}=\left\{P_{i} \mid 0 \leq P_{i} \leq P_{\max }\right\}, \quad \text { for all } i .
$$

Here, we assume that the maximum power of each secondary user is very large, i.e., $P_{\max } \geq 2 w_{S} N / C_{0}$, where $C_{0}$ is the unit price for secondary-user transmit power [see (16)], and $w_{S}$ is the weight factor for secondary-user utility [see (17)].

The primary user transmits its data in Phases I and II and collects revenue in Phases II and III. The utility of the primary user is the sum of data transmitted and revenue collected, as

$$
U_{P}=w_{P} f_{P}\left(R_{P}\right)+\sum_{k=1}^{K} C_{0} P_{i}
$$

where $R_{P}=\alpha \beta R_{P}^{I}+\alpha(1-\beta) R_{P}^{I I}$ is the amount of primaryuser data transmitted, $w_{P}$ is a weight factor, $C_{0}$ is the unit price for secondary-user power, and $f_{P}(x)$ is the satisfaction function of the primary user with respect to data transmission. Since the primary user always has some data to send, it requires a minimum data rate. Naturally, we choose $f_{P}(x)=\ln (x)$, $x \geq 0$. The negative value for very small $x$ serves as a penalty that forces the primary user to achieve a minimum data rate. 
From the shape of $f_{P}(x)$, we know that at the initial stage, the primary user is enthusiastic about data transmission. After a period of transmission, even a great increase in the data transmission can only result in a small increase in the satisfaction. Note that we assume the primary user always has some data to send in each time slot. If the primary user has no data to send, it will provide all the time and spectrum to the secondary users and merely collect revenues. This way, the primary user is in fact serving as a service provider rather than as a service user. We exclude this case in the following analysis, while focusing on the case when the primary user is also a spectrum user.

Since the primary user is rational and selfish, it aims to maximize $U_{P}$ by controlling the lengths of the three phases and selecting secondary users to participate in the game. By adjusting weight $w_{P}$, the primary users can trade off between data transmission and revenue collection. This could be related to the content type that the primary user is transmitting. If the primary user is transmitting a high-resolution video, it may assign a large number to $w_{P}$. That is, the primary user currently values data transmission much more than revenue collection. To maximize $U_{P}$, the primary user can simply set $\alpha=1$ and $\beta=1$ (i.e., there are no Phases II and III). If the primary user is surfing the Internet and is delay tolerant, it may assign $w_{P}$ a small number to make revenue collection more important for maximizing $U_{P}$.

Selected secondary users transmit their data during Phases II and III and make a one-time payment to the primary user in each time slot. The utility of the secondary user is given by

$$
U_{S_{i}}=w_{S} f_{S}\left(R_{S_{i}}\right)-C_{0} P_{i}
$$

where $R_{S_{i}}=\alpha(1-\beta) R_{S_{i}}^{I I}+(1-\alpha) R_{S_{i}}^{I I I}, f_{S}(x)$ is the satisfaction function of the secondary user, and $w_{S}$ is the weight factor. As in prior work [8], we assume identical $w_{S}$ for all the secondary users to simplify notation. The solution could be easily extended to the case of heterogeneous $w_{S}$ values. Since the essence of CR is to opportunistically exploit an underutilized spectrum, we choose $f_{S}(x)=x$, indicating that the secondary users operate in a best effort manner. By assigning a large value to $w_{S}$, the secondary user cares more about its data transmission. On the contrary, if a small value is assumed for $w_{S}$, the secondary user is more concerned about the payment to the primary user. The weight $w_{S}$ allows a secondary user to trade off between data transmission and payment.

Therefore, we define a Stackelberg game, with players, their roles, strategies [see (14) and (15)], and utilities [see (16) and (17)] specified. We provide a thorough analysis of the game with respect to the existence and uniqueness of the Stackelberg equilibrium and optimal strategies in Section IV.

\section{B. Discussion}

From a secondary user's point of view, it prefers to transmit more data while keeping the cost as low as possible. If there are fewer players, the DoF can be increased. Since the DoF is a pre$\log$ factor [see (12) and (13)], transmitting with a larger power when the DoF is high is definitely a better choice. At the same time, since the primary user will not participate in Phase III, the DoF could be further increased with one less player in this phase. Once the one-time payment is made, the secondary users can transmit during both Phases II and III and prefer longer periods for these phases. To sum up, with the unit price fixed, the secondary users favor fewer players and longer duration of Phase II or Phase III, preferably Phase III.

As the leader, the primary user has the advantage of making a tradeoff between data transmission and revenue collection. In Phase I, the primary user's transmission rate is high. More primary user data could be transmitted if Phase I is longer. In Phase II, the primary user could collect revenue while transmitting data, although at a lower data rate. With more secondary users selected, more players are paying the primary user, which is helpful in maximizing its utility. However, if too many secondary users are selected, the DoF for each player will also be drastically decreased. Under this situation, there is no revenue since no one could transmit, and thus, no one would pay. Therefore, $K$ should be carefully decided. In summary, the primary users' strategy should consider the tradeoff between data transmission and revenue collection. Since in Phase II, the primary user can transmit while collecting revenue, and the choices of $\alpha, \beta$, and $K$ are dependent, the primary-user decision is highly complicated.

\section{Performance Analysis and Solution Strategy}

Here, we analyze the formulated Stackelberg game to find a strategy set for the primary user and secondary users such that no one could gain by a unilateral change of strategy. Let $\vec{P}^{*}$ be the vector of secondary-user power and $\vec{P}_{-i}^{*}=\vec{P}^{*} \backslash P_{i}$. We first define Stackelberg equilibrium as follows.

Definition 1 (Stackelberg Equilibrium): A strategy set $\left\{\alpha^{*}\right.$, $\left.\beta^{*}, K^{*}, \vec{P}^{*}\right\}$ is a Stackelberg equilibrium of the game defined in Section III if the following conditions are satisfied:

1) $U_{P}\left(\alpha^{*}, \beta^{*}, K^{*}, \vec{P}^{*}\right) \geq U_{P}\left(\alpha, \beta, K, \vec{P}^{*}\right)$ for all $\alpha \in[0,1]$, $\beta \in[0,1]$, and $K \in\left[0, K_{T}\right]$.

2) $U_{S_{i}}\left(P_{i}^{*}, \vec{P}_{-i}^{*}, \alpha^{*}, \beta^{*}, K^{*}\right) \geq U_{S_{i}}\left(P_{i}, \vec{P}_{-i}^{*}, \alpha^{*}, \beta^{*}, K^{*}\right)$ for all $\alpha \in[0,1], \beta \in[0,1], K \in\left[0, K_{T}\right]$, and $i \in[1, K]$.

Using the backward induction method [19], we prove the uniqueness of the Stackelberg equilibrium and derive the unique Stackelberg equilibrium (and the optimal strategy) for the game defined in Section III in the remainder of this section.

\section{A. Secondary-User Utility Maximization}

From (17), the utility of the secondary user is given by

$$
\begin{aligned}
U_{S_{i}}\left(P_{i}\right)=w_{S} f_{S}\left(R_{S_{i}}\right)-C_{0} P_{i} \\
=w_{S}\left[\alpha(1-\beta) d_{I I} \log \left(P_{i} / N_{0}\right)+(1-\alpha)\right. \\
\left.\quad \times d_{I I I} \log \left(P_{i} / N_{0}\right)\right]-C_{0} P_{i} .
\end{aligned}
$$

To maximize its utility, the secondary user solves the following maximization problem:

$$
\max _{0 \leq P_{i} \leq P_{\max }} U_{S_{i}}\left(P_{i}\right)
$$


For given $\alpha$ and $\beta, U_{S_{i}}\left(P_{i}\right)$ is a concave function of $P_{i}$. Setting $\left(d U_{S_{i}} / d P_{i}\right)=0$, we derive the unique maximizer of (19), which is

$$
P_{i}^{*}=\frac{w_{S} \alpha(1-\beta) d_{I I}+w_{S}(1-\alpha) d_{I I I}}{C_{0}} .
$$
have

Since $0 \leq \alpha \leq 1,0 \leq \beta \leq 1$, and $d_{I I} \leq d_{I I I} \leq 2 N$, we

$$
P_{i}^{*} \leq w_{S} d_{I I I} / C_{0} \leq 2 w_{S} N / C_{0} \leq P_{\max }
$$

indicating that the $P_{i}^{*}$ given in (20) is a feasible solution. It follows that the maximum utility of the secondary user is

$$
U_{S_{i}}^{*}=Y \log \left(Y /\left[2 C_{0} N_{0}\right]\right), \quad i \in[1, K]
$$

where $Y=w_{S}\left[\alpha\left(d_{I I}-d_{I I I}\right)-\alpha \beta d_{I I}+d_{I I I}\right]$.

Since $U_{S_{i}}^{*}$ is a monotonically increasing function of $Y$, and $d_{I I} \leq d_{I I I}$, it can be verified that $U_{S_{i}}^{*}$ is a monotonically decreasing function of $\alpha$ and $\beta$. Since $\alpha(1-\beta) \geq 0$ and $(1-$ $\alpha) \geq 0, U_{S_{i}}^{*}$ is a monotonically increasing function of $d_{I I}$ and $d_{I I I}$. From a secondary user's perspective, the best scenario is $\alpha=0, \beta=0$, and $K=3$, i.e., the entire time slot is Phase III with the minimum number of followers. The selected secondary users enjoy the highest data rate during the entire time slot. The primary user can only collect revenue from the three secondary users. This is consistent with our conjectures in Section III-B. Note that this is the best case as only the secondary users are concerned. From later discussions, we can see that the primary user, who also tries to maximize its utility, may set the parameters in part but not completely according to the secondary users' preference.

\section{B. Primary-User Utility Maximization}

Given the optimal strategies of all the secondary users, we substitute $f_{P}\left(R_{P}\right)$ and $P_{i}^{*}$ into (16). It follows that

$$
\begin{aligned}
U_{P}(\alpha, \beta, K)= & w_{P} \ln \left[\alpha \beta R_{P}^{I}+\alpha(1-\beta) R_{P}^{I I}\right] \\
& +K w_{S}\left[\alpha(1-\beta) d_{I I}+(1-\alpha) d_{I I I}\right] .
\end{aligned}
$$

The primary user solves the following problem to maximize its utility:

$$
\max _{0 \leq \alpha \leq 1,0 \leq \beta \leq 1,3 \leq K \leq K_{T}} U_{P}\left(\alpha, \beta, K, \vec{P}^{*}\right) .
$$

Maximization of the primary-user utility is more complicated. We examine the problem for different parameter ranges and derive the local maximizer in each range. The global optimum is found by comparing the local maximizers. This is similar to finding the maximum element in a matrix: We first find the largest element in each column; then, we compare these elements from different columns to find the largest element in the matrix. Without loss of generality, we assume that $w_{P}=$ $w_{S} .{ }^{2}$ The analysis can be extended to the case when $w_{P} \neq w_{S}$.

\footnotetext{
${ }^{2}$ There are some special cases for $w_{P}$ and $w_{S}$. For instance, if $w_{P}=\infty$, the primary user will not lease a spectrum to secondary users, which is a degenerated case. We focus on the general cases when $w_{P}=w_{S}$.
}

a) Case I-When $K_{T} \geq(2 N-1)$ :

When $3 \leq K \leq(2 N-1)$ : First, let us consider $K \in$ $[3,2 N-1] . U_{P}$ can be rewritten as follows:

$$
\begin{gathered}
U_{P}=w_{P} \ln \left\{\log (\mathrm{SNR})\left[\alpha \beta N+\alpha(1-\beta)\left\lfloor\frac{2 N}{K+2}\right\rfloor\right]\right\} \\
+K w_{S}\left[\alpha(1-\beta)\left\lfloor\frac{2 N}{K+2}\right\rfloor+(1-\alpha)\left\lfloor\frac{2 N}{K+1}\right\rfloor\right] .
\end{gathered}
$$

Note that $K$ and $\beta$ are dependent variables. If $\beta=1$, there is no Phase II. We next consider $\beta=1$ and $\beta \in[0,1)$.

Case $(a)-\beta=1$ : We denote the utility of the primary user as $U_{P}^{0}$ in this case, which is given by

$$
\begin{aligned}
U_{P}^{0} & =w_{P} \ln (\alpha N \log (\mathrm{SNR}))+K w_{S}(1-\alpha)\left\lfloor\frac{2 N}{K+1}\right\rfloor \\
& \leq w_{P} \ln (\alpha N \log (\mathrm{SNR}))+w_{S}(1-\alpha) \frac{2 N}{1+\frac{1}{K}} \\
& \leq w_{P} \ln (\alpha N \log (\mathrm{SNR}))+w_{S}(1-\alpha)(2 N-1) .
\end{aligned}
$$

The two equalities hold true when $K=2 N-1$. We then have the following optimization problem:

$$
\max _{0 \leq \alpha \leq 1} U_{P}^{0}(\alpha, 1,2 N-1)
$$

where $\quad U_{P}^{0}(\alpha, 1,2 N-1)=w_{P} \ln (\alpha N \log (\mathrm{SNR}))+w_{S}(2 N-1)$ $(1-\alpha)$. Since $U_{P}^{0}$ is concave with respect to $\alpha$, problem (27) can be solved with convex programming [25]. $U_{P}^{0}$ achieves its maximum when $\alpha=(1 / 2 N-1)$, and its maximum is given by

$$
\begin{aligned}
U_{P}^{* 0}\left(\frac{1}{2 N-1}, 1,2 N-1\right)=w_{P} \ln ( & \left.\frac{N}{2 N-1} \log (\mathrm{SNR})\right) \\
& +w_{S}(2 N-2) .
\end{aligned}
$$

Case (b) $-\beta \in[0,1)$ : Relaxing $K$ to a continuous variable and ignoring the floor functions, we have

$$
\begin{aligned}
\frac{\partial U_{P}}{\partial K}=w_{P}\left\{-\frac{2(1-\beta)}{(K+2)^{2} \beta+2(K+2)(1-\beta)}\right. & \\
& \left.+\frac{4 N \alpha(1-\beta)}{(K+2)^{2}}+\frac{2 N(1-\alpha)}{(K+1)^{2}}\right\} .
\end{aligned}
$$

The first item is irrelevant to $\alpha$, whereas the last two items are linear in $\alpha$. If for both $\alpha=0$ and $\alpha=1,\left(\partial U_{P} / \partial K\right) \geq 0$ holds true for any $\beta$, then for any $0 \leq \alpha \leq 1$ and $0 \leq \beta<1$, $\left(\partial U_{P} / \partial K\right) \geq 0$.

We prove this conjecture as follows. When $\alpha=0$, we have

$$
\begin{aligned}
\frac{\partial U_{P}}{\partial K} & =\frac{2 N w_{P}}{(K+1)^{2}}-\left(\frac{w_{P}}{K+2}\right)\left(\frac{2(1-\beta)}{\beta K+2}\right) \\
& \geq \frac{2 N w_{P}}{(K+1)^{2}}-\frac{w_{P}}{K+2} \geq w_{P}\left[\frac{1}{K+1}-\frac{1}{K+2}\right] \geq 0 .
\end{aligned}
$$

The first inequality occurs because $\beta \geq 0$, such that $(2(1-$ $\beta) / \beta K+2) \leq 1$. The second inequality is due to the fact that $2 N \geq(K+1)$. 
When $\alpha=1$, we have

$$
\begin{aligned}
\frac{\partial U_{P}}{\partial K} & =w_{P} \frac{(1-\beta)}{(K+2)^{2}}\left[4 N-(K+2) \frac{2}{\beta K+2}\right] \\
& \geq w_{P} \frac{(1-\beta)}{(K+2)^{2}}[4 N-(K+2)] \geq 0 .
\end{aligned}
$$

The first inequality is due to $\beta \geq 0$, such that $(2 / \beta K+2) \leq 1$. The second inequality is due to the fact that $2 N \geq(K+1)$.

Therefore, if we treat $K$ as a continuous variable and ignore the floor functions, $U_{P}$ is a monotonically increasing function of $K$. To maximize $U_{P}$, we should have $K=2 N-1$. Now, consider $K$ as an integer and take the floor functions into account. We show that we should have $K=2 N-2$ in this case.

If $K=2 N-1$, denote the utility of the primary user in this case as $U_{P}^{1}$. Since $\lfloor(2 N / K+2)\rfloor=0$ and $\lfloor(2 N / K+1)\rfloor=1$, we have

$$
U_{P}^{1}=w_{P} \ln (\alpha \beta N \log (\mathrm{SNR}))+w_{S}(2 N-1)(1-\alpha) .
$$

It can be verified that $U_{P}^{1}$ is an increasing function of $\beta$ for $\beta \in[0,1)$. Thus, we have $U_{P}^{1}<U_{P}^{0}$. It follows that

$$
U_{P}^{* 1}<U_{P}^{* 0}
$$

Given (30), we no longer need to examine the maximization of $U_{P}^{1} ; K=2 N-1$ can be discarded for $\beta \in[0,1)$. As a matter of fact, we could see from later discussion that $\max _{\alpha, \beta} U_{P}(\alpha, \beta, 2 N-1)<\max _{\alpha, \beta} U_{P}(\alpha, \beta, 2 N-2)$. Since $K=2 N-1$ is excluded, we only need to consider $K \leq$ $2 N-2$. Rewrite (25) as

$$
\begin{aligned}
U_{P}= & w_{P} \ln \left(N \beta+(1-\beta)\left\lfloor\frac{2 N}{K+2}\right\rfloor\right) \\
& +K w_{S}\left[\alpha(1-\beta)\left\lfloor\frac{2 N}{K+2}\right\rfloor+(1-\alpha)\left\lfloor\frac{2 N}{K+1}\right\rfloor\right] \\
& +w_{P} \ln (\alpha \log (\mathrm{SNR})) .
\end{aligned}
$$

Defining $f_{1}(K)=\ln (N \beta+(1-\beta)\lfloor(2 N / K+2)\rfloor)$ and $f_{2}(K)=$ $K[\alpha(1-\beta)\lfloor(2 N / K+2)\rfloor+(1-\alpha)\lfloor(2 N / K+1)\rfloor]$, we have the following Lemma for $f_{2}(K)$.

Lemma 1: $\arg \max _{K \in[3,2 N-2]} f_{2}(K)=2 N-2$.

Proof: For the first item in $f_{2}(K)$, we have

$$
K\left\lfloor\frac{2 N}{K+2}\right\rfloor \leq K \frac{2 N}{K+2} \leq 2 N-\frac{4 N}{K+2} \leq 2 N-2 .
$$

The equalities hold true only for $K=2 N-2$. For the second item in $f_{2}(K)$, if there is no constraint on $K, K\lfloor(2 N / K+$ 1) $\rfloor=0$ for $K>2 N-1$. For $K \leq 2 N-1$, we have

$$
K\left\lfloor\frac{2 N}{K+1}\right\rfloor \leq K \frac{2 N}{K+1} \leq 2 N-\frac{2 N}{K+1} \leq 2 N-1 .
$$

The equalities hold true only for $K=2 N-1$. When the constraint $K \leq 2 N-2$ is enforced, if $K=2 N-2, K\lfloor(2 N / K+$ 1) $\rfloor=2 N-2$. Since $K\lfloor(2 N / K+1)\rfloor$ can only be integers, and $2 N-2$ is only 1 less than $2 N-1,2 N-2$ is the largest number we can have for $K\lfloor(2 N / K+1)\rfloor$ when $K \leq 2 N-2$.
Since both $K\lfloor(2 N / K+2)\rfloor$ and $K\lfloor(2 N / K+1)\rfloor$ are maximized at $K=2 N-2, f_{2}(K)$ attains its maximum at $K=$ $2 N-2$.

Lemma 2: For $K^{\prime} \in(N-2,2 N-2), \quad U_{P}\left(\alpha, \beta, K^{\prime}\right)<$ $U_{P}(\alpha, \beta, 2 N-2)$.

Proof: For $K^{\prime} \in(N-2,2 N-2)$, we always have $\lfloor(2 N /$ $\left.\left.K^{\prime}+2\right)\right\rfloor=1$. When $K=2 N-2,\lfloor(2 N / K+2)\rfloor=1$. Thus, $f_{1}\left(K^{\prime}\right)=f_{1}(2 N-2)$. On the other hand, $K^{\prime}\left\lfloor\left(2 N / K^{\prime}+2\right)\right\rfloor<$ $(2 N-2)\lfloor(2 N /(2 N-2)+2)\rfloor$. For $K^{\prime} \in(N-2,2 N-2)$, it can be verified that $K^{\prime}\left\lfloor\left(2 N / K^{\prime}+1\right)\right\rfloor \leq(2 N-2)\lfloor(2 N /$ $(2 N-2)+1)\rfloor$ for $N \geq 2$. We thus have $f_{2}\left(K^{\prime}\right)<f_{2}(2 N-$ $2)$. Summing up $f_{1}(K)$ and $f_{2}(K)$, we have $U_{P}\left(\alpha, \beta, K^{\prime}\right)<$ $U_{P}(\alpha, \beta, 2 N-2)$.

The insight from Lemma 2 is that if $2 N$ is not divisible by $K+2$, this $K$ value is not useful for the optimization and can be safely discarded. We have the following corollary.

Corollary 2.1: Assume $2 N$ is divisible by $\left(K_{1}+2\right),\left(K_{2}+2\right)$, $\ldots,\left(K_{n}+2\right)$, and $K_{1}>K_{2}>\cdots>K_{n}$, for any $K^{\prime \prime} \in$ $\left(K_{2}, K_{1}\right), \ldots, K^{\prime \prime} \overbrace{}^{n} \in\left(K_{n}, K_{n-1}\right)$, we have

$$
U_{P}(\alpha, \beta, K^{\prime \prime} \overbrace{}^{\prime})<U_{P}\left(\alpha, \beta, K_{i-1}\right), \quad \forall i=2, \ldots, n .
$$

According to Corollary 2.1, to find the value of $K$ that maximizes $U_{P}$, we only need to consider the $K$ values such that $2 N$ is divisible by $K+2$.

Lemma 3: If $K_{0}=N-2$ is feasible, it follows that $U_{P}(\alpha, \beta, 2 N-2)>U_{P}(\alpha, \beta, N-2)$.

Proof: $K_{0}=N-2$ is feasible if $K_{0} \geq 3$. It follows that $N \geq 5$ in this case. Therefore, we have

$$
\left\lfloor\frac{2 N}{K_{0}+1}\right\rfloor=\left\lfloor\frac{2 N}{N-1}\right\rfloor=2+\left\lfloor\frac{2}{N-1}\right\rfloor=2 .
$$

It follows that

$$
\begin{aligned}
& U_{P}(2 N-2)-U_{P}(N-2) \\
& \quad=w_{P}\left[\ln \left(\frac{N \beta+(1-\beta)}{N \beta+2(1-\beta)}\right)+2(1-\alpha \beta)\right] \\
& \geq w_{P}\left[\ln \left(\frac{N \beta+(1-\beta)}{N \beta+2(1-\beta)}\right)+2(1-\beta)\right] .
\end{aligned}
$$

The inequality is because $U_{P}(2 N-2)-U_{P}(N-2)$ is a monotonically decreasing function of $\alpha$. For $\beta=0, U_{P}(2 N-$ 2) $-U_{P}(N-2)=w_{P}[2-\ln (2)]>0$. For $\beta \in(0,1)$, define $f_{3}(N)=\ln (N \beta+(1-\beta) / N \beta+2(1-\beta))$ and treat $N$ as a continuous variable. We have

$$
\frac{\partial f_{3}(N)}{\partial N}=\frac{\beta}{N \beta+(1-\beta)}-\frac{\beta}{N \beta+2(1-\beta)}>0
$$

which indicates that $f_{3}(N)$ is a strictly monotonically increasing function of $N$. Since, currently, $N \geq 5$, we have $f_{3}(N)>$ $f_{3}(1)=-\ln (2-\beta)$. That is

$U_{P}(2 N-2)-U_{P}(N-2)>w_{P}[-\ln (2-\beta)+2(1-\beta)]$. 
Define $f_{4}(\beta)=-\ln (2-\beta)+2(1-\beta)$. Since $\left(\partial^{2} f_{4} / \partial \beta^{2}\right)=$ $\left(1 /(2-\beta)^{2}\right)>0, f_{4}(\beta)$ is a convex function. The domain $\{\beta \mid \beta \in(0,1)\}$ is also a convex set. Suppose $\beta$ can be equal to 0 and 1 . Solving the following problem:

$$
\min _{0 \leq \beta \leq 1}-\ln (2-\beta)+2(1-\beta)
$$

we have $\min _{\beta \in[0,1]} f_{4}(\beta)=0$, and the minimum is achieved at $\beta=1$. We conclude that $f_{4}(\beta)>0$ for $\beta \in(0,1)$.

It follows that $U_{P}(\alpha, \beta, 2 N-2)-U_{P}(\alpha, \beta, N-2) \geq$ $w_{P}\left[f_{3}(N)+2(1-\beta)\right]>w_{P}\left[f_{3}(1)+2(1-\beta)\right]>0$. The proof is completed.

Lemma 4: Consider $K_{1}, K_{2}, \ldots, K_{n}$, such that $2 N$ is divisible by $K_{1}+2, K_{2}+2, \ldots$, and $K_{n}+2$, and if $\left(2 N / K_{1}+\right.$ $2)=3,\left(2 N / K_{2}+2\right)=4, \ldots,\left(2 N / K_{n}+2\right)=N$, it follows that $U_{P}(\alpha, \beta, 2 N-2)>U_{P}\left(\alpha, \beta, K_{i}\right), i=1,2, \ldots, n$.

Proof: For $K_{1}$, we have

$$
\begin{aligned}
& U_{P}(N-2)-U_{P}\left(K_{1}\right) \\
& \quad=w_{P}\left[\ln \left(\frac{N \beta+2(1-\beta)}{N \beta+3(1-\beta)}\right)+2(1-\alpha \beta)\right] \\
& \quad \geq w_{P}\left[\ln \left(\frac{N \beta+2(1-\beta)}{N \beta+3(1-\beta)}\right)+2(1-\beta)\right] \\
& >w_{P}\left[\ln \left(\frac{N \beta+(1-\beta)}{N \beta+2(1-\beta)}\right)+2(1-\beta)\right]>0 .
\end{aligned}
$$

The first inequality is due to the fact that $U_{P}(N-2)-$ $U_{P}\left(K_{1}\right)$ is a monotonically decreasing function of $\alpha$. The second inequality is due to $\ln (N \beta+2(1-\beta) / N \beta+3(1-$ $\beta))>\ln (N \beta+(1-\beta) / N \beta+2(1-\beta))$ for $\beta \in[0,1)$, and the last inequality is proved in Lemma 3 . Thus, we have

$$
U_{P}(2 N-2)>U_{P}(N-2)>U_{P}\left(K_{1}\right) .
$$

For $K_{2}$, we have

$$
\begin{aligned}
& U_{P}\left(K_{1}\right)-U_{P}\left(K_{2}\right) \\
& \quad=w_{P}\left[\ln \left(\frac{N \beta+3(1-\beta)}{N \beta+4(1-\beta)}\right)+2(1-\alpha \beta)\right] \\
& \quad \geq w_{P}\left[\ln \left(\frac{N \beta+3(1-\beta)}{N \beta+4(1-\beta)}\right)+2(1-\beta)\right] \\
& \quad>w_{P}\left[\ln \left(\frac{N \beta+2(1-\beta)}{N \beta+3(1-\beta)}\right)+2(1-\beta)\right]>0 .
\end{aligned}
$$

Repeat the given steps for $K_{3}, \ldots, K_{n}$. The proof is completed.

Theorem 1: When $K_{T} \geq 2 N-1,3 \leq K \leq 2 N-1$, and $0 \leq \beta<1, U_{P}$ is maximized when $K=2 N-2$.

Proof: We have shown in Lemma 3 that if $K_{0}$ exists, $U_{P}(2 N-2)>U_{P}\left(K_{0}\right)$. We have also shown in Lemma 4 that if $K_{i}, i=1, \ldots, n$ exists, $U_{P}(2 N-2)>U_{P}\left(K_{i}\right)$. In addition, considering Corollary 2.1, K=2N-2 is the maximizer.

Substitute $K=2 N-2$ into (31), we have

$$
\begin{aligned}
U_{P}(2 N-2)=w_{P}\{\ln [(\alpha \log (\mathrm{SNR}))(N \beta+(1-\beta))]\} \\
+w_{S}(2 N-(2)(1-\alpha \beta) .
\end{aligned}
$$

We next divide the range of $\alpha$ into three ranges and examine each of them in the following.

Case $(a)-\alpha \in[0,1 / 2 N]$ : Denoting the utility of the primary user in this case as $U_{P}^{2}$, we have

$$
\begin{aligned}
\frac{\partial U_{P}^{2}}{\partial \beta} & =w_{P} \frac{N-1}{N \beta+(1-\beta)}-w_{S}(2 N-2) \alpha \\
& \geq w_{P}\left[\frac{N-1}{(N-1) \beta+1}-\frac{N-1}{N}\right] \\
& >w_{P}\left[\frac{N-1}{(N-1)+1}-\frac{N-1}{N}\right]=0 .
\end{aligned}
$$

The first inequality occurs because $\left(\partial U_{P}^{2} / \partial \beta\right)$ is a monotonically decreasing function of $\alpha$, and the second inequality is due to $\beta<1$. Hence, $U_{P}$ is a monotonically increasing function of $\beta$. For $\alpha \in[0,(1 / 2 N)]$, we have $U_{P}^{2}<$ $w_{P} \ln [N \alpha \log (\mathrm{SNR})]+w_{S}(2 N-2)(1-\alpha)<U_{P}^{0} \leq U_{P}^{* 0}$.

This case can be safely discarded.

Case $(b)-\alpha \in[(1 / 2), 1]$ : Denoting the utility of the primary user in this case as $U_{P}^{3}$, we have

$$
\begin{aligned}
\frac{\partial U_{P}^{3}}{\partial \beta} & =w_{P} \frac{N-1}{N \beta+(1-\beta)}-w_{S}(2 N-(2) \alpha \\
& \leq w_{P}\left[\frac{N-1}{(N-1) \beta+1}-(N-1)\right] \leq 0 .
\end{aligned}
$$

The first inequality occurs because $\left(\partial U_{P}^{3} / \partial \beta\right)$ is a monotonically decreasing function of $\alpha$, and the second inequality is due to $\beta \geq 0$. Hence, $U_{P}$ is a nonincreasing function of $\beta$. Letting $\beta=0$, we have the following maximization problem:

$$
\max _{\frac{1}{2} \leq \alpha \leq 1} U_{P}^{3}=w_{P} \ln (\alpha \log (\mathrm{SNR}))+w_{S}(2 N-2) .
$$

Since $U_{P}^{3}$ is now a monotonically increasing function of $\alpha$, letting $\alpha=1$, we have

$U_{P}^{* 3}(1,0,2 N-2)=w_{P} \ln (\log (\mathrm{SNR}))+w_{S}(2 N-2)$.

For $N \geq 2$, we have $U_{P}^{* 3}-U_{P}^{* 0}=w_{P} \ln (2 N-1 / N)>0$. Recall that $U_{P}^{* 1}<U_{P}^{* 0}$, as previously stated. It follows that $U_{P}^{* 1}<U_{P}^{* 3}$. The case of $K=2 N-1$ can be also safely discarded.

Case (c) $-\alpha \in((1 / 2 N),(1 / 2))$ : Denote the utility of the primary user in this case as $U_{P}^{4} . U_{P}^{4}$ is a concave function of $\beta$. Letting $\left(\partial U_{P}^{4} / \partial \beta\right)=0$, we have $\hat{\beta}=(1 / 2 \alpha-1)(1 / N-1)$. Since $\alpha>(1 / 2 N), \hat{\beta}<1$. Since $\alpha<(1 / 2), \hat{\beta}>0$. Hence, $\hat{\beta}=((1 / 2 \alpha)-1)(1 / N-1)$ is feasible. Substitute $\hat{\beta}$ into $U_{P}^{4}$, we have

$$
U_{P}^{4}=w_{P} \ln \left(\frac{1}{2} \log (\mathrm{SNR})\right)+w_{S}(2 N+2 \alpha-3) .
$$

Since $U_{P}^{4}$ is a monotonically increasing function of $\alpha, U_{P}^{* 4}<$ $w_{P} \ln ((1 / 2) \log (\mathrm{SNR}))+w_{S}(2 n-2)<U_{P}^{* 0}<U_{P}^{* 3}$. Therefore, we have the following lemma. 
Lemma 5: For $K_{T} \geq 2 N-1$ and $K \leq 2 N-1, U_{P}$ achieves its maximum when $\alpha=1, \beta=0, K=2 N-2$, and the maximum value is given by (34).

a) When $K>(2 N-1)$ : For $K>2 N-1$, we always have $\lfloor(2 N / K+2)\rfloor=0$ and $\lfloor(2 N / K+1)\rfloor=0$. Denote the utility of the primary user in this case as $U_{P}^{5}$, we have

$$
U_{P}^{5}=w_{P} \ln (N \alpha \beta \log (\mathrm{SNR})) .
$$

Obviously, $U_{P}^{5}$ is a monotonically increasing function of $\alpha$ and $\beta$. Hence, the maximum is achieved when $\alpha=1$ and $\beta=1$. Thus

$$
U_{P}^{* 5}=w_{P} \ln (N \log (\mathrm{SNR})) .
$$

Note that under this condition, there are no Phases II and III. There is no spectrum leasing, and the transmission rates of all the secondary users are 0 .

Comparing $U_{P}^{* 5}$ with $U_{P}^{* 3}$, we have

$$
\begin{aligned}
U_{P}^{* 3}-U_{P}^{* 5} & =-w_{P} \ln (N)+w_{S}(2 N-(2) \\
& >w_{P}[(2 N-(2)-N] \geq 0 .
\end{aligned}
$$

The first inequality is due to $\ln (x)<x$ for $x>0$, and the second inequality is due to $N \geq 2$. Therefore, $U_{P}^{* 3}>U_{P}^{* 5}$. The implication of (36) is that leasing a spectrum to secondary users is helpful in maximizing the utility of the primary user. ${ }^{3}$

Compared with Lemma 5, we summarize the given analysis as a lemma as follows.

Lemma 6: For $K_{T} \geq 2 N-1, U_{P}$ achieves its maximum when $\alpha=1, \beta=0, K=2 N-2$, and the maximum of $U_{P}$ is given in (34).

2) Case II-When $K_{T}=(2 N-2)$ : It can be readily concluded that the conclusion given in Section IV-B1 still holds. Hence, we finally have the following theorem.

Theorem 2: When $K_{T} \geq 2 N-2, U_{P}$ is maximized when $\alpha=1, \beta=0, K=2 N-2$, and the maximum of $U_{P}$ is given in (34).

Note that when $K=2 N-2, d_{I I}=1, d_{I I I}=1$. Theorem 2 indicates that when there are plenty of secondary users, to maximize the primary user's utility, we should select $2 N-2$ out of them so that each of the selected secondary user can have exactly one interference-free channel. Since $\alpha=1$ and $\beta=0$, there is no Phase I and Phase III. To maximize the primary-user utility, there is no need for the primary user to use MIMO transmission alone. Transmitting data with distributed interference alignment while collecting revenue from spectrum leasing is the best strategy for the primary user.

3) Case III-When $3 \leq K_{T} \leq(2 N-3)$ : Here, we consider the case when $3 \leq K_{T} \leq 2 N-3$. Hence, the number of antennas must satisfy $2 N-3 \geq 3$, which indicates that $N \geq 3$.

For simplicity, we assume that $2 N$ is divisible by both $K_{T}+$ 2 and $K_{T}+1$. That is, $\left\lfloor\left(2 N / K_{T}+2\right)\right\rfloor=\left(2 N / K_{T}+2\right)$, and $\left\lfloor\left(2 N / K_{T}+1\right)\right\rfloor=\left(2 N / K_{T}+1\right)$. Using similar arguments as in Section IV-B1, to maximize $U_{P}$, we should let $K=K_{T}$.

\footnotetext{
${ }^{3}$ One may note that if $w_{P} \gg w_{S}$, the inequality does not hold. However, as we noted before, we focus on the generic case where $w_{P}=w_{S}$.
}

Given the strategies of all the secondary users, the primary user tries to maximize its own utility by solving the following problem:

$$
\max _{0 \leq \alpha \leq 1,0 \leq \beta \leq 1} U_{P}(\alpha, \beta) .
$$

Plug in $K_{T}$ and $P_{i}^{*}$, we have

$$
\begin{aligned}
U_{P}(\alpha, \beta)= & w_{P} \ln \left\{\alpha\left[\beta\left(R_{P}^{I}-R_{P}^{I I}\right)+R_{P}^{I I}\right]\right\} \\
& +K_{T} w_{S}\left\{\alpha\left[(1-\beta) d_{I I}-d_{I I I}\right]+d_{I I I}\right\} .
\end{aligned}
$$

We also assume that $w_{P}=w_{S}$. To find the maximum, we divide the $\alpha$-axis into three adjacent intervals: $[0,(1 / 2 N)]$, $\left[(1 / 2 N),\left(K_{T}+2 / 4 N\right)\right]$ and $\left[\left(K_{T}+2 / 4 N\right), 1\right]$. Note that for $K_{T} \geq 3,(1 / 2 N)<\left(K_{T}+2 / 4 N\right)$.

Case $(a)-0 \leq \alpha \leq(1 / 2 N)$ : Denote the utility of the primary user as $U_{P}^{6}$, we have

$$
\begin{aligned}
\frac{\partial U_{P}^{6}}{\partial \beta} & =w_{P} \frac{R_{P}^{I}-R_{P}^{I I}}{\beta R_{P}^{I}+(1-\beta) R_{P}^{I I}}-K_{T} w_{S} \alpha d_{I I} \\
& \geq w_{P}\left[\frac{d_{I}-d_{I I}}{d_{I}}-K_{T} \alpha d_{I I}\right] \\
& =w_{P}\left[\frac{K_{T}}{K_{T}+2}-K_{T} \alpha \frac{2 N}{K_{T}+2}\right] \geq 0
\end{aligned}
$$

where the first inequality is due to $\max _{\beta \in[0,1]} \beta d_{I}+(1-$ $\beta) d_{I I}=d_{I}$, and the second inequality is due to $\alpha \leq(1 / 2 N)$.

Hence, for $0 \leq \alpha \leq(1 / 2 N), U_{P}(\alpha, \beta)$ is a monotonically increasing function of $\beta$. That is, $U_{P}(\alpha, \beta) \leq U_{P}(\alpha, 1)$. To maximize the utility, the primary user solves the following problem:

$$
\max _{0 \leq \alpha \leq \frac{1}{2 N}} U_{P}^{6}(\alpha, 1)=w_{P} \ln \left(\alpha R_{P}^{I}\right)+K_{T} w_{S}(1-\alpha) d_{I I I} .
$$

Using convex programming, it can be found that $U_{P}$ achieves its maximum when $\alpha=(1 / 2 N)$. Moreover, the maximum value is

$$
\begin{aligned}
U_{P}^{* 6}\left(\frac{1}{2 N}, 1\right) & =w_{P} \ln \left(\frac{R_{P}^{I}}{2 N}\right)+K_{T} w_{S}\left(\frac{2 N-1}{2 N}\right) d_{I I I} \\
& =w_{P} \ln \left(\frac{\log (\mathrm{SNR})}{2}\right)+K_{T} w_{S} \frac{2 N-1}{K_{T}+1}
\end{aligned}
$$

Case $(b)-\left(K_{T}+2 / 4 N\right) \leq \alpha \leq 1$ : Denote the utility of the primary user as $U_{P}^{7}$, we have

$$
\begin{aligned}
\frac{\partial U_{P}^{7}}{\partial \beta} & =w_{P}\left[\frac{d_{I}-d_{I I}}{\beta d_{I}+(1-\beta) d_{I I}}-K_{T} \alpha d_{I I}\right] \\
& \leq w_{P}\left[\frac{d_{I}-d_{I I}}{d_{I I}}-K_{T} \alpha d_{I I}\right] \\
& =w_{P}\left[\frac{K_{T}}{2}-K_{T} \alpha \frac{2 N}{K_{T}+2}\right] \leq 0
\end{aligned}
$$

where the first inequality is due to $\min _{\beta \in[0,1]} \beta d_{I}+(1-$ $\beta) d_{I I}=d_{I I}$, and the last inequality is due to $\alpha \geq\left(K_{T}+\right.$ $2 / 4 N)$. 
Thus, for $\left(K_{T}+2 / 4 N\right) \leq \alpha \leq 1, U_{P}^{7}(\alpha, \beta)$ is a monotonically decreasing function of $\beta$, which indicates that $U_{P}^{7}(\alpha, \beta) \leq$ $U_{P}^{7}(\alpha, 0)$. To maximize utility, the primary user solves the following problem:

$$
\begin{aligned}
\max _{\frac{K_{T}+2}{4 N} \leq \alpha \leq 1} U_{P}(\alpha, 0)= & w_{P} \ln \left(\alpha R_{P}^{I I}\right) \\
& +K_{T} w_{S}\left[\alpha d_{I I}+(1-\alpha) d_{I I I}\right] .
\end{aligned}
$$

Using convex programming, it can be found that $U_{P}$ achieves its maximum when $\alpha=\left(\left(K_{T}+1\right)\left(K_{T}+2\right) / 2 N K_{T}\right)$. Notice that, since we assume that $2 N$ is divisible by both $K_{T}+1$ and $K_{T}+2, \quad\left(\left(K_{T}+1\right)\left(K_{T}+2\right) / 2 N\right) \leq 1$. That is, $\alpha=\left(\left(K_{T}+1\right)\left(K_{T}+2\right) / 2 N K_{T}\right)<1$. On the other hand, $\left(\left(K_{T}+1\right)\left(K_{T}+2\right) / 2 N K_{T}\right)>\left(K_{T}+2 / 4 N\right)$; hence, $\left(\left(K_{T}+1\right)\left(K_{T}+2\right) / 2 N K_{T}\right)$ is a feasible point. The maximum value is given by

$$
\begin{array}{r}
U_{P}^{* 7}\left(\frac{\left(K_{T}+1\right)\left(K_{T}+2\right)}{2 N K_{T}}, 0\right)=w_{P} \ln \left[\log (\mathrm{SNR}) \frac{K_{T}+1}{K_{T}}\right] \\
+K_{T} w_{S}\left(\frac{2 N}{K_{T}+1}-\frac{1}{K_{T}}\right) .
\end{array}
$$

Case $(c)-(1 / 2 N) \leq \alpha \leq\left(K_{T}+2 / 4 N\right)$ : Denote the utility of the primary user as $U_{P}^{8}$. For any fixed $\alpha, U_{P}^{8}$ is a concave function with respect to $\beta$. We could maximize $U_{P}^{8}$ by first maximizing it with respect to $\beta$ and then with respect to $\alpha$. We have

$$
\frac{\partial U_{P}^{8}}{\partial \beta}=w_{P} \frac{R_{I}-R_{I I}}{\beta R_{I}+(1-\beta) R_{I I}}-K_{T} w_{S} \alpha d_{I I} .
$$

Setting $\left(\partial U_{P}^{8} / \partial \beta\right)=0$ results in

$$
\beta=\frac{1}{K_{T} \alpha d_{I I}}-\frac{d_{I I}}{d_{I}-d_{I I}} .
$$

Since $\alpha \geq(1 / 2 N), \beta \leq\left(1 / K_{T}\left(2 N / K_{T}+2\right)(1 / 2 N)\right)-((2 N /$ $\left.\left.K_{T}+2\right) / N-\left(2 N / K_{T}+2\right)\right)=1 ; \quad \alpha \leq\left(K_{T}+2 / 4 N\right), \quad \beta \geq$ $\left(1 / K_{T}\left(2 N / K_{T}+2\right)\left(K_{T}+2 / 4 N\right)\right)-\left(2 / K_{T}\right)=0$. Hence, the value of $\beta$ given by (46) is a feasible point. Under this condition, we have

$$
\begin{aligned}
U_{P}^{8}= & w_{P} \ln \left(\frac{w_{P}\left(R_{P}^{I}-R_{P}^{I I}\right)}{K_{T} w_{S} d_{I I}}\right)+K_{T} w_{S} \\
& \times\left\{d_{I I I}-\frac{w_{P}}{K_{T} w_{S}}+\alpha\left[\left(1+\frac{R_{I I}}{R_{I}-R_{I I}}\right) d_{I I}-d_{I I I}\right]\right\} \\
= & w_{P} \ln \left(\frac{\log (\mathrm{SNR})}{2}\right)+w_{P} \alpha \frac{2 N}{\left(K_{T}+1\right)} \\
& -w_{P}+K_{T} w_{P} d_{I I I}
\end{aligned}
$$

which is monotonically increasing function of $\alpha$. When $\alpha=$ $\left(K_{T}+2 / 4 N\right)$, the maximum is attained. Plug the value of $\alpha$ into (46), we have $\beta=0$. Hence, the maximum value is given by

$U_{P}^{* 8}\left(\frac{K_{T}+2}{4 N}, 0\right)=w_{P}\left[\ln \left(\frac{\log (\mathrm{SNR})}{2}\right)+\frac{K_{T}(4 N-1)}{2\left(K_{T}+1\right)}\right]$.
It can be readily concluded that $U_{P}^{* 6}<U_{P}^{* 8}$, we only need to compare $U_{P}^{* 7}$ with $U_{P}^{* 8}$, then we could find the maximum value of $U_{P}$. We have

$$
U_{P}^{* 7}-U_{P}^{* 8}=w_{P}\left[\ln \left(2+\frac{2}{K_{T}}\right)-\frac{1}{2}-\frac{1}{2\left(K_{T}+1\right)}\right] .
$$

Denote $f_{5}\left(K_{T}\right)=\ln \left(2\left(K_{T}+1 / K_{T}\right)\right)-\left(K_{T}+2 / 2\left(K_{T}+\right.\right.$ 1)). Consider $K_{T}$ as a continuous variable, we have

$$
\frac{\partial f_{5}}{\partial K_{T}}=\frac{-\left(K_{T}+2\right)}{2 K_{T}\left(K_{T}+1\right)^{2}}<0 .
$$

Hence, $f_{5}\left(K_{T}\right)$ is a monotonically decreasing function of $K_{T}$, which means that $f_{5}\left(K_{T}\right)>f_{5}(+\infty)$. Therefore, we have

$$
U_{P}^{* 7}-U_{P}^{* 8}>w_{P}\left[\ln (2)-\frac{1}{2}\right]=0.193>0 .
$$

Since $U_{P}^{* 7}>U_{P}^{* 6}$ and $U_{P}^{* 7}>U_{P}^{* 8}$, we readily have the following theorem.

Theorem 3: For $3 \leq K_{T} \leq 2 N-3, U_{P}$ achieves its maximum when $\alpha=\left(\left(K_{T}+1\right)\left(K_{T}+2\right) / 2 N K_{T}\right), \beta=0, K=$ $K_{T}$, and the maximum of $U_{P}$ is given by (44).

It would still be interesting to compare $U_{P}^{* 7}$ with $U_{P}^{* 5}$ for which there is no spectrum leasing. We have

$$
\begin{aligned}
& U_{P}^{* 7}-U_{P}^{* 5} \\
& =w_{P}\left[\ln \left(\frac{K_{T}+1}{K_{T}}\right)+2 N \frac{K_{T}}{K_{T}+1}-1-\ln (N)\right] \\
& >w_{P}\left[2 N \frac{K_{T}}{K_{T}+1}-1-\ln (N)\right] \\
& \geq w_{P}\left[\frac{3}{2} N-1-\ln (N)\right]>w_{P}\left[\frac{1}{2} N-1\right]>0
\end{aligned}
$$

where the first inequality is due to $\ln (1+x)>0$ for $x>0$, the second inequality is because $\left(K_{T} / K_{T}+1\right)$ is a monotonically increasing function of $K_{T}$, the third inequality is due to $\ln (x)<$ $x$ for $x>0$, and the last inequality is due to $N \geq 3$.

This indicates that even with an insufficient number of secondary users, leasing a spectrum to the secondary users is still beneficial for the primary user to increase its utility.

\section{Unique Stackelberg Equilibrium}

We now summarize the analysis in Section IV-A and B. The unique Stackelberg equilibrium of the game defined in Section III is given in the following theorem.

Theorem 4: The unique Stackelberg equilibrium is given by

$$
\begin{aligned}
& \left(\alpha^{*}, \beta^{*}, K^{*}\right) \\
& \quad= \begin{cases}(1,0,2 N-2), & \text { if } K_{T} \geq 2 N-2 \\
\left(\frac{\left(K_{T}+1\right)\left(K_{T}+2\right)}{2 N K_{T}}, 0, K_{T}\right), & \text { if } 3 \leq K_{T} \leq 2 N-3\end{cases}
\end{aligned}
$$

$P_{i}^{*}$

$$
=\left[w_{S} \alpha^{*}\left(1-\beta^{*}\right) d_{I I}+w_{S}\left(1-\alpha^{*}\right) d_{I I I}\right] / C_{0}, \text { for all } i .
$$

Since we can rewrite (54) as $P_{i}^{*}=w_{S}\left[\alpha\left(d_{I I}-d_{I I I}\right)-\right.$ $\left.\alpha \beta d_{I I}+d_{I I I}\right] / C_{0}$ and $d_{I I} \geq d_{I I I}, P_{i}^{*}$ is a monotonically 


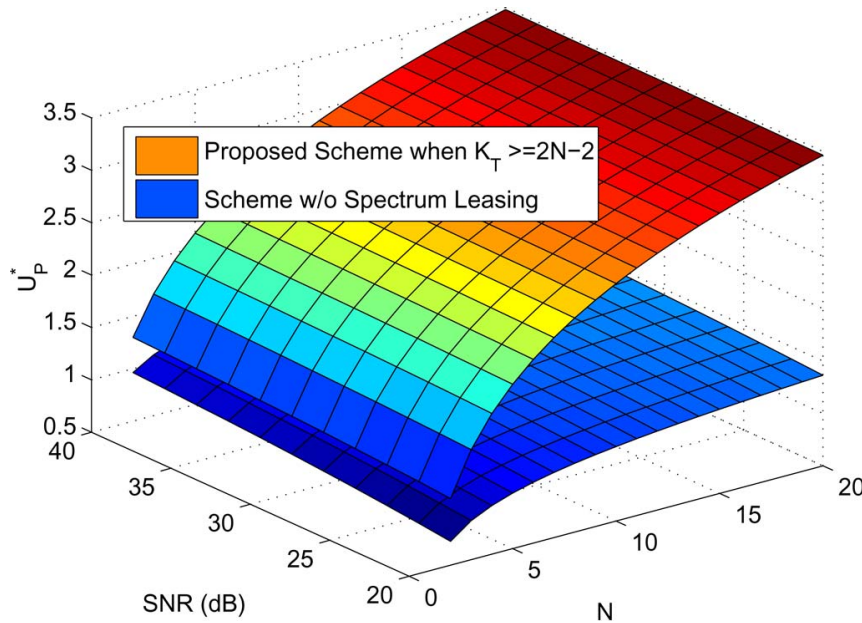

Fig. 2. Utility of the primary user in log scale.

decreasing function of $\alpha$ and $\beta$. On the other hand, $P_{i}^{*}$ is a monotonically increasing function of $d_{I I}$ and $d_{I I I}$, indicating that $P_{i}^{*}$ is a monotonically decreasing function of $K$. The secondary users will adjust their transmitter power in light of $\alpha, \beta$, and $K$. The best scenario for them is $\alpha=0, \beta=0$, and $K=3$, for which there is only Phase III with the fewest players.

Knowing the optimal strategies of the secondary users, the primary user will set $\alpha=1, \beta=0$, and $K=2 N-2$ when there is a sufficient number of secondary users. Each selected secondary user has exactly one interference-free channel, and there is only Phase II in the time slot. In this case, the primary user can collect as much revenue as possible while keeping a relatively low-rate data transmission. The secondary users' claim is satisfied in part. If there are not as many secondary users as needed, the primary user will set the parameters carefully according to (53). Under this condition, the primary user selects all the secondary users, discards Phase I, and makes a tradeoff between Phase II and Phase III according to how many secondary users are there in the system.

\section{Simulation Study}

Simulations are conducted to validate the performance of the proposed scheme. We first compare the proposed scheme with a scheme without spectrum leasing to demonstrate the benefits of spectrum leasing. We then compare the proposed scheme with the cooperative scheme presented in [8] to demonstrate the efficacy of MIMO and distributed interference alignment.

\section{A. With or Without Spectrum Leasing}

We first consider the case when there is a sufficient number of secondary users, i.e., $K_{T} \geq 2 N-2$, since in many realworld applications, there are usually more secondary users than the number of antennas at each node. In Fig. 2, we plot the primary-user utility $U_{P}^{*}$ versus the number of antennas $N$ and SNR. In the simulation, the weight factors are $w_{P}=w_{S}=$ 0.8 . The noise spectral density is $N_{0}=0.1$. The unit price is $C_{0}=0.001$. Note that the maximum utility of the primary user without spectrum leasing is given in (35). It is shown in Fig. 2 that there is a huge gap between the proposed scheme and the

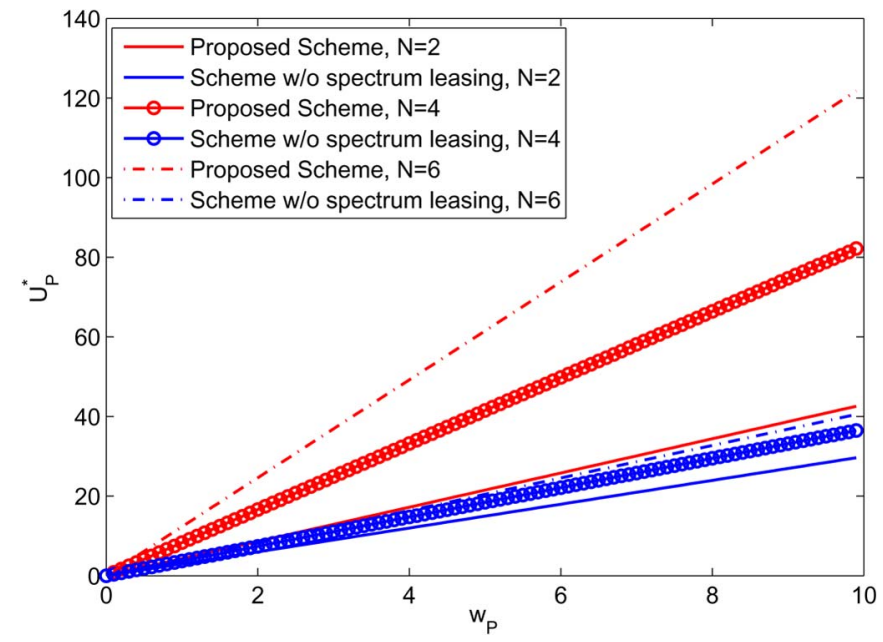

Fig. 3. Utility of the primary user when $K_{T} \geq 2 N-2$.

scheme without spectrum leasing. Note that the utility increase due to SNR is less obvious than that due to $N$, since the impact of SNR is diminished by the logarithm functions in (10) and (11). This clearly indicates that under the same setting, leasing a spectrum to secondary users can greatly improve the primaryuser utility. In addition, note that, from (36), the utility of the proposed scheme is strictly larger than that of no spectrum leasing, for any feasible values of $w_{P}, N$, and SNR.

In Fig. 3, we examine the impact of weight $w_{P}$ on the primary-user utility $U_{P}^{*}$. We plot the results with or without spectrum leasing and for $N=2,4$, and 6 . It can be seen that when $w_{P}$ is increased, the gap between the proposed scheme and the scheme without spectrum leasing becomes larger. Although with increased $w_{P}$, the primary user emphasizes more on data transmission, the revenue is still increased at a higher speed with spectrum leasing. The gap also becomes larger when the number of antennas for each node is increased. This is also because the revenue increases faster with spectrum leasing than the no-leasing scheme as $N$ is increased.

We then consider the case of an insufficient number of secondary users. In the simulation, there are $K_{T}=3$ secondary users. The number of antennas is $N=20$. We plot the primaryuser utility for the proposed scheme and the no-spectrumleasing scheme in Fig. 4. There is also a big gain achieved by the proposed scheme. This is consistent with our previous discussions. In this case, the primary user should still lease its spectrum to secondary users to maximize its own utility.

\section{B. With or Without Distributed MIMO and Distributed Interference Alignment}

Next, we compare our proposed scheme with the cooperative scheme in [8]. To make fair comparisons, replace the satisfaction function $f_{P}\left(R_{P}\right)=\left(1 / 1+e^{-a\left(R_{P}-R_{0}\right)}\right)$ in [8] with $f_{P}\left(R_{P}\right)=\ln \left(R_{P}\right)$. We first derive an upper bound of the utility of the primary user (denoted by $U_{P}^{9}$ ) in [8] using our notation and then compare our proposed scheme with the upper bound. Thus

$$
U_{P}^{9}=w_{P} \ln \left(R_{P}\right)+\frac{w_{S}(1-\alpha)(K-1)}{\sum_{i}\left(\frac{1}{R_{S_{i}}}\right)}
$$




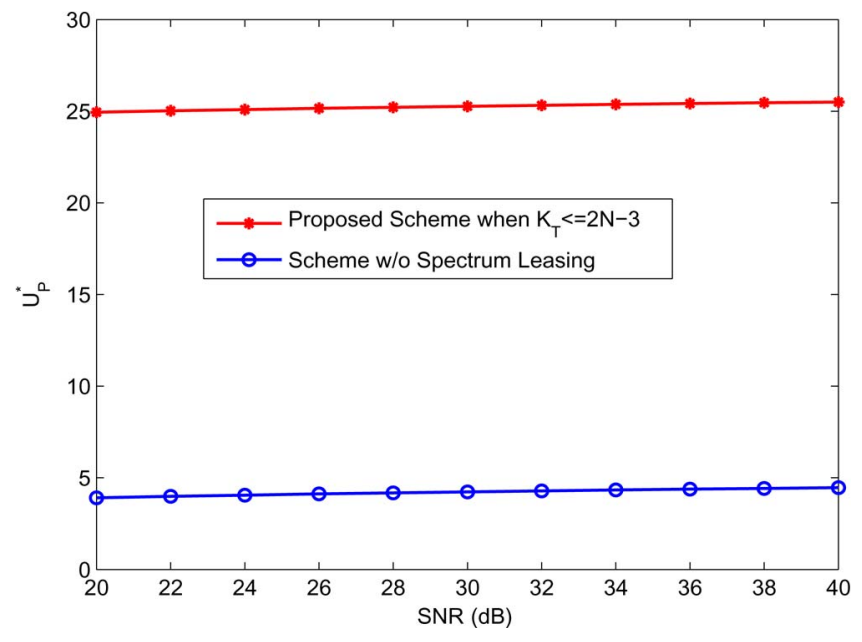

Fig. 4. Utility of the primary user when $K_{T} \leq 2 N-3$.

where $R_{P}=\min \left\{\alpha \beta R_{P S}, \alpha(1-\beta) R_{S P}\right\}, R_{P S}=\log \left(1+\left(\min _{i} \mid\right.\right.$ $\left.\left.\left.h_{P S, i}\right|^{2} P / N_{0}\right)\right), R_{S P}=\log \left(1+\left(\left.h_{P}\right|^{2} P / N_{0}\right)+\sum_{i}\left(\min _{i}\left|h_{S P, i}\right|^{2} P /\right.\right.$ $\left.\left.N_{0}\right)\right), R_{S_{i}}=\log \left(1+\left(\left|h_{S_{i}}\right|^{2} P / N_{0}\right)\right)$, and all the $h$ 's are channel states. Since

$$
\left\{\begin{aligned}
R_{P} & =\min \left\{\alpha \beta R_{P S}, \alpha(1-\beta) R_{S P}\right\} \\
& \leq \alpha \frac{R_{P S} R_{S_{P}}}{R_{P S}+R_{S P}}<\alpha R_{P S} \leq \alpha \log \left(1+\frac{P}{N_{0}}\right) . \\
R_{S_{i}} & \leq \log \left(1+\frac{P}{N_{0}}\right) .
\end{aligned}\right.
$$

It follows from (55) and (56) that $U_{P}^{9}<w_{P} \ln [\alpha \log (1+$ SNR $)]+\left(w_{S}(1-\alpha)(K-1) / K\right) \log (1+$ SNR $)$. Denote $f_{6}(\alpha)=$ $\ln [\alpha \log (1+\mathrm{SNR})]+((1-\alpha)(K-1) / K) \log (1+\mathrm{SNR})$. For $\mathrm{SNR} \geq 3, f_{6}(\alpha)$ is maximized at $\hat{\alpha}=(K /(K-1) \log (1+$ SNR)). Since we consider a high-SNR region, the condition of $\mathrm{SNR} \geq 3$ is easily satisfied. Plugging in $\hat{\alpha}$, we have

$$
U_{P}^{9}<w_{P} \ln \left(\frac{K}{K-1}\right)+w_{S}\left[\frac{K-1}{K} \log (1+\mathrm{SNR})-1\right]
$$

indicating that the utility of the cooperative scheme is upper bounded by $w_{P} \ln (K / K-1)+w_{S}[(K-1 / K) \log (1+$ SNR) -1$]$.

In Fig. 5, we plot the simulation results for the proposed scheme, the cooperative scheme, and the no-spectrum-leasing scheme. Recall that in [8], all the primary user and secondary users are equipped with a single antenna. To make fair comparisons, we choose $K$, which is the number of secondary users selected, as the variable in the simulation. In the simulations, since the number of antennas must satisfy $\lfloor(2 N / K+2)\rfloor \geq 1$, as the value of $K$ varies, we set $N=\lceil(K+2 / 2)\rceil$. Hence, we are actually comparing the lower bound of our proposed scheme with the upper bound of the cooperative scheme. It is shown in Fig. 5 that both spectrum leasing schemes outperform the nospectrum-leasing scheme. Furthermore, the proposed scheme outperforms the cooperative scheme with considerable gains. Such gains justify the efficacy of MIMO and distributed interference alignment, which greatly enhances the overall system capacity.

Finally, we compare the proposed scheme with the cooperative scheme in [8] in terms of aggregate secondary-user utility

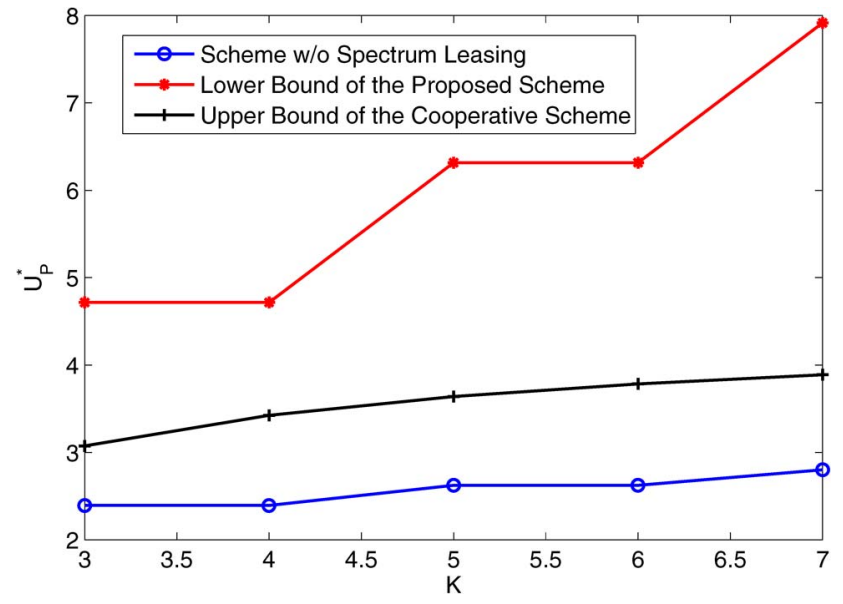

Fig. 5. Comparison of the proposed scheme with the cooperative scheme.

and average secondary-user utility. We first derive an upper bound for the secondary-user utility in [8] and then compare it with the secondary-user utility achieved with the proposed scheme with an identical number of selected secondary users and identical transmission power. Note that, under the scenario of no spectrum leasing, the secondary-user utility is always 0 . Therefore, we do not include this case in the comparison.

From Theorem 4, we obtain the maximum utility for each secondary user and the aggregate maximum utility for all the secondary users as

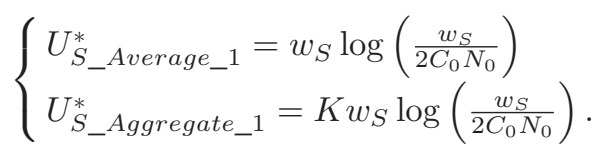

The utility for each secondary user in [8] is given by

$$
\max _{c_{i}} u_{i}\left(c_{i}\right)=\max _{c_{i}}\left\{\frac{w_{S}(1-\alpha) c_{i} R_{i}}{\sum_{j} c_{j}}-c_{i}\right\}
$$

where $R_{i}=\log \left(1+\left(\left|h_{S_{i}}\right|^{2} P_{S} / N_{0}\right)\right)$. Since we assume perfect channel and consider a high SNR, $R_{i} \approx R=\log \left(P_{S} / N_{0}\right)$. The maximum is achieved at

$c_{i}^{*}=w_{S}(1-\alpha)(K-1)\left[\sum_{j} \frac{1}{R_{j}}-\frac{K-1}{R_{i}}\right]\left(\sum_{j} \frac{1}{R_{j}}\right)^{-2}$.

Letting $X_{i}=(K-1)\left[\sum_{j}\left(1 / R_{j}\right)-\left(K-1 / R_{i}\right)\right] /\left(\sum_{j}\left(1 / R_{j}\right)\right)^{2}$, we have $c_{i}^{*}=w_{S}(1-\alpha) X_{i}$. The maximum aggregate secondaryuser utility denoted by $U_{S_{-}}^{*}$ Aggregate_2 is derived as follows:

$$
\begin{aligned}
U_{S \_ \text {Aggregate_2 }}^{*} & =\sum_{i} u_{i}\left(c_{i}^{*}\right) \leq w_{S}\left[\frac{\sum_{i} X_{i} R_{i}}{X_{i}}-\sum_{i} X_{i}\right] \\
& <w_{S} \frac{\sum_{i} X_{i} R_{i}}{X_{i}} \approx w_{S} R=w_{S} \log \left(\frac{P_{S}}{N_{0}}\right)
\end{aligned}
$$

where the first inequality is due to $0 \leq \alpha \leq 1$, and the second inequality is due to $X_{i}>0$. 


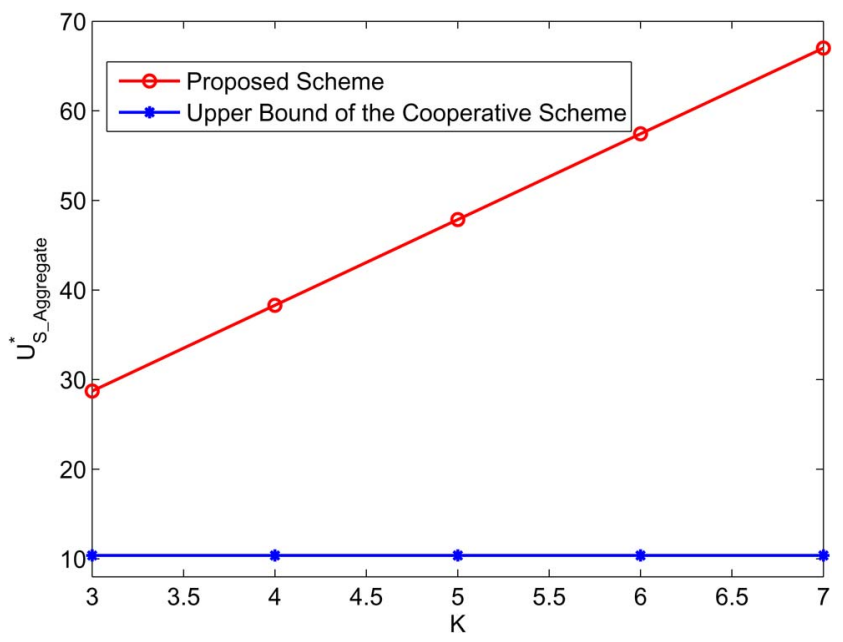

Fig. 6. Aggregate secondary-user utility comparison of the proposed scheme with the cooperative scheme.

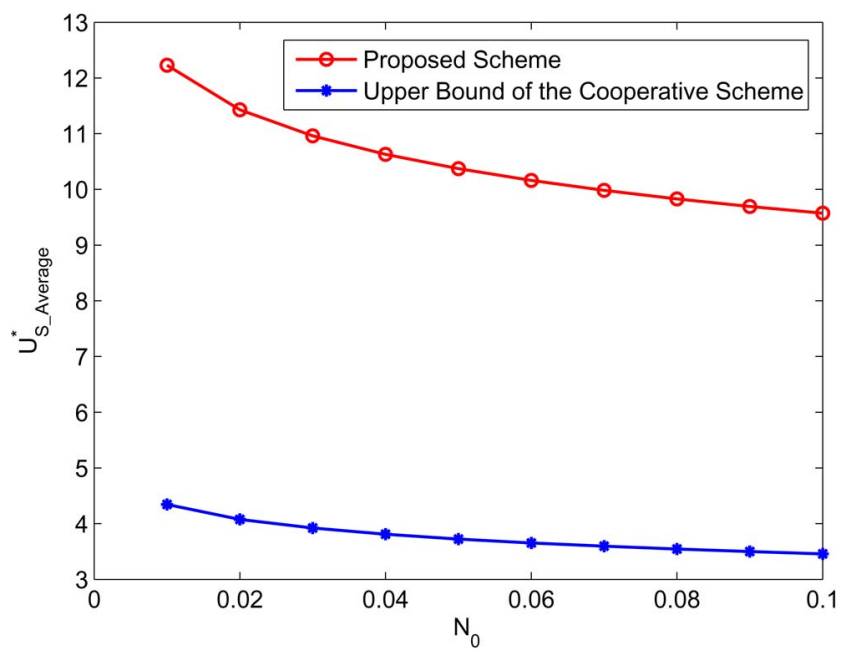

Fig. 7. Average secondary-user utility comparison of the proposed scheme with the cooperative scheme.

Substituting $P_{i}^{*}$ from Theorem 4, we have

$$
\left\{\begin{array}{l}
U_{S \_ \text {Aggregate_2 }}^{*}=w_{S} \log \left(\frac{w_{S}}{C_{0} N_{0}}\right) \\
U_{S \_ \text {Average } \_2}^{*}=\frac{w_{S}}{K} \log \left(\frac{w_{S}}{C_{0} N_{0}}\right) \leq \frac{w_{S}}{3} \log \left(\frac{w_{S}}{C_{0} N_{0}}\right)
\end{array}\right.
$$

where the inequality is due to $K \geq 3$.

It is shown in Figs. 6 and 7 that the proposed scheme outperforms the cooperative scheme, in both cases of the average secondary-user utility and the total secondary-user utility.

\section{CONCLUSION}

In this paper, we have investigated the behaviors of the primary user and secondary users in a MIMO CR network. We proposed a three-phase cooperative spectrum leasing scheme with distributed interference alignment. The system was modeled as a Stackelberg game. With backward induction, we derived the unique Stackelberg equilibrium. Through rigorous analysis, we found the best strategies for the primary user and secondary users under a broad range of conditions and parameters and discussed practical implications. We also found that leasing a spectrum to secondary users is always helpful in enhancing the primary-user utility. Simulation results demonstrated that the proposed scheme outperformed a nospectrum-leasing scheme and a cooperative scheme from prior work. For future work, it would be interesting to implement the proposed scheme in a programmable wireless platform and test its performance in a practical setting.

\section{REFERENCES}

[1] Y. $\mathrm{Xu}$ and S. Mao, "Distributed interference alignment in $\operatorname{cog}$ nitive radio networks," in Proc. IEEE ICCCN, Nassau, Bahamas, Jul./Aug. 2013, pp. 1-7.

[2] "Spectrum policy task force," Washington, DC, USA, Rep. ET Docket no. 02-135, Nov. 2002.

[3] Y. Zhao, S. Mao, J. Neel, and J. H. Reed, "Performance evaluation of cognitive radios: Metrics, utility functions, and methodologies," Proc. IEEE, vol. 97, no. 4, pp. 642-659, Apr. 2009.

[4] A. Goldsmith, S. A. Jafar, I. Maric, and S. Srinivasa, "Breaking spectrum gridlock with cognitive radios: An information theoretic perspective," Proc. IEEE, vol. 97, no. 5, pp. 894-914, May 2009.

[5] J. Mietzner, R. Schober, L. Lampe, W. H. Gerstacker, and P. A. Hoeher, "Multiple-antenna techniques for wireless communications-A comprehensive literature survey," IEEE Commun. Surveys Tuts., vol. 11, no. 2, pp. 87-105, 2nd Quart., 2009.

[6] V. R. Cadambe and S. A. Jafar, "Interference alignment and degrees of freedom of the $K$-user interference channel," IEEE Trans. Inf. Theory, vol. 54, no. 8, pp. 3425-3441, Aug. 2008.

[7] K. Gomadam, T. Gou, V. R. Cadambe, and S. A. Jafar, "A distributed numerical approach to interference alignment and applications to wireless interference networks," IEEE Trans. Inf. Theory, vol. 57, no. 6, pp. 33093322, Jun. 2011.

[8] J. Zhang and Q. Zhang, "Stackelberg game for utility-based cooperative cognitive radio networks," in Proc. ACM MobiHoc, New Orleans, LA, USA, May 2011, pp. 23-31.

[9] A. A. Daoud, T. Alpcan, S. Agarwal, and M. Alanyali, "A Stackelberg game for pricing uplink power in wide-band cognitive radio networks," in Proc. IEEE CDC, Cancun, Mexico, Dec. 2008, pp. 1422-1427.

[10] Y. Xiao, G. Bi, and D. Niyato, "Distributed optimization for cognitive radio networks using Stackelberg game," in Proc. IEEE ICCS, Singapore, Nov. 2010, pp. 77-81.

[11] D. Niyato, E. Hossain, and Z. Han, "Dynamics of multiple-seller and multiple buyer spectrum trading in cognitive radio networks: A game theoretic modeling approach," IEEE Trans. Mobile Comput., vol. 8, no. 8, pp. 1009-1022, Aug. 2009.

[12] D. Hu and S. Mao, "Cooperative relay with interference alignment for video over cognitive radio networks," in Proc. IEEE INFOCOM, Orlando, FL, USA, Mar. 2012, pp. 2014-2022.

[13] C. M. Yetis, T. Gou, S. A. Jafar, and A. H. Kayran, "On feasibility of interference alignment in MIMO interference networks," IEEE Trans. Signal Process., vol. 58, no. 9, pp. 4771-4782, Sep. 2010.

[14] Y. Xu and S. Mao, "On interference alignment in multi-user OFDM systems," in Proc. IEEE GLOBECOM, Anaheim, CA, USA, Dec. 2012, pp. 5339-5344.

[15] S. Gollakota, S. D. Perli, and D. Katabi, "Interference alignment and cancellation," in Proc. ACM SIGCOMM, Barcelona, Spain, Aug. 2009, pp. 159-170.

[16] L. E. Li, R. Alimi, D. Shen, H. Viswanathan, and Y. R. Yang, "A general algorithm for interference alignment and cancellation in wireless networks," in Proc. IEEE INFOCOM, San Diego, CA, USA, Mar. 2010, pp. $1-9$.

[17] M. Amir, A. El-Keyi, and M. Nafie, "Opportunistic interference alignment for multiuser cognitive radio," in Proc. IEEE ITW, Dublin, Ireland, Aug./Sep. 2010, pp. 1-5.

[18] H. Zhou, T. Ratnarajah, and Y. Liang, "On secondary network interference alignment in cognitive radio," in Proc. IEEE DySPAN, Aachen, Germany, May 2011, pp. 637-641.

[19] D. Fudenberg and J. Tirole, Game Theory. Cambridge, MA, USA: MIT Press, 1993. 
[20] K.-H. Park and M.-S. Alouini, "Alternate MIMO AF relaying networks with interference alignment: Spectral efficient protocol and linear filter design," IEEE Trans. Veh. Technol., vol. 62, no. 2, pp. 914-920, Feb. 2013.

[21] T.-W. Tang, M.-K. Chen, and H.-F. Lu, "Improving the DMT performance for MIMO communication with linear receivers," IEEE Trans. Veh. Technol., vol. 62, no. 3, pp. 1189-1200, Mar. 2013.

[22] K. T. Truong and R. W. Heath, Jr., "Joint transmit precoding for the relay interference broadcast channel," IEEE Trans. Veh. Technol., vol. 62, no. 3 , pp. 1201-1215, Mar. 2013.

[23] D. Tse and P. Viswanath, Fundamentals of Wireless Communication. Cambridge, U.K.: Cambridge Univ. Press, 2005.

[24] Q. Zhao and B. Sadler, "A survey of dynamic spectrum access," IEEE Signal Process. Mag., vol. 24, no. 3, pp. 79-89, May 2007.

[25] S. Boyd and L. Vandenberghe, Convex Optimization. Cambridge, U.K.: Cambridge Univ. Press, 2004.

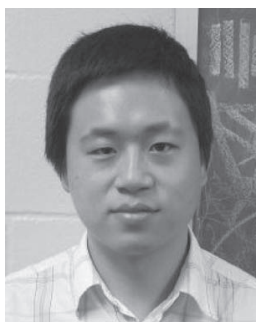

Yi Xu (S'11) received the B.S. degree in electronic information engineering from the University of Electronic Science and Technology of China, Chengdu, China, in 2007 and the M.S. degrees in electronic engineering from Tsinghua University, Beijing, China, in 2010 . He is currently working toward the Ph.D. degree with the Department of Electrical and Computer Engineering, Auburn University, Auburn, AL, USA.

His research interests include optimization, game theory, multiple-input multiple-output, orthogonal frequency-division multiplexing, interleave-division multiple access, and cognitive radio networks.

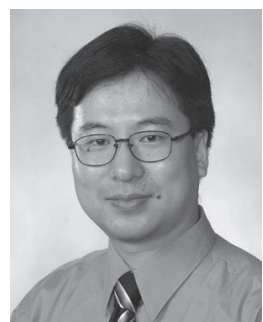

Shiwen Mao (S'99-M'04-SM'09) received the $\mathrm{Ph} . \mathrm{D}$. degree in electrical and computer engineering from Polytechnic University, Brooklyn, NY, USA (now Polytechnic Institute of New York University), in 2004.

From 1997 to 1998 , he was a research staff member with IBM China Research Laboratory, Beijing, China. From 2003 to 2006, he was a Postdoctoral Research Fellow/Research Scientist with the Bradley Department of Electrical and Computer Engineering, Virginia Tech, Blacksburg, VA, USA. He is currently the McWane Associate Professor with the Department of Electrical and Computer Engineering, Auburn University, Auburn, AL, USA. He is a coauthor of TCP/IP Essentials: A Lab-Based Approach (Cambridge University Press, 2004) and a coeditor of several other books. He is the holder of one U.S. patent. His research interests include wireless networks and multimedia communications, with current focus on cognitive radio, small cell, $60-\mathrm{GHz}$ millimeter-wave networks, free-space optical networks, and smart grid.

Dr. Mao coreceived the IEEE International Communications Conference (ICC) 2013 Best Paper Award. He received the 2013 IEEE ComSoc Multimedia Communications Technical Committee (MMTC) Outstanding Leadership Award and was named the 2012 Exemplary Editor of IEEE COMMUNICATIONS SuRVEys AND Tutorials. He received the McWane Endowed Professorship from the Samuel Ginn College of Engineering for the Department of Electrical and Computer Engineering, Auburn University, in August 2012. He received the U.S. National Science Foundation Faculty Early Career Development Award (CAREER) in 2010. He coreceived the 2004 IEEE Communications Society Leonard G. Abraham Prize in the Field of Communications Systems and the Best Paper Runner-up Award at the 2008 Conference on Heterogeneous Networking for Quality, Reliability, Security, and Robustness. He also received Auburn Alumni Council Research Awards for Excellence-Junior Award and two Auburn Author Awards in 2011. He is on the Editorial Board of the IEEE TRANSACTIONS ON WiRELESS COMMUNICATIONS, the IEEE INTERNET OF THINGS JOURNAL, the IEEE COMMUNICATIONS SURVEYS AND TUtorials, the ElSEVIER AD Hoc Networks Journal, the WiLEY INTERNATIONAL JOURNAL OF COMMUNICATION SYSTEMS, and the EAI ENDORSED TRANSACTIONS ON MOBILE COMMUNICATIONS AND APPLICATIONS. For the period 2012 to 2014, he is the Director of E-Letter of MMTC of the IEEE Communications Society. He is the Technical Program Vice Chair for Information Systems of the IEEE 34th International Conference on Computer Communications (INFOCOM 2015); a Symposium Cochair for many conferences, including the IEEE ICC, the IEEE Global Communications Conference, the IEEE International Conference on Computer Communications and Networks, and the Joint IEEE International Conference on Industrial Technology and IEEE Southeastern Symposium on System Theory, among others; he has held various positions on the organizing committees of many conferences. 